\title{
Basin-Scale Tidal Measurements using Acoustic Tomography
}

by

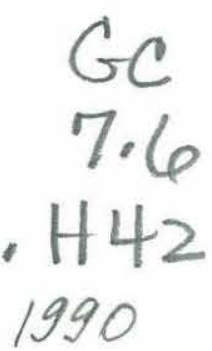

Robert Hugh Headrick

B.S. Chem. Eng., Oklahoma State University (1983)

Submitted in partial fulfillment of the

requirements for the degrees of

OCEAN ENGINEER

and

MASTER OF SCIENCE IN OCEAN ENGINEERING

at the
MASSACHUSETTS INSTITUTE OF TECHNOLOGY
and the

\section{WOODS HOLE OCEANOGRAPHIC INSTITUTION}

September 1990

(C) Robert Hugh Headrick, 1990

The author hereby grants to the United States Government, MIT, and WHOI permission to reproduce and

to distribute copies of this thesis document in whole or in part.

Signature of Author $\ldots \ldots, \ldots, \ldots, \ldots, \ldots, \ldots, \ldots, \ldots, \ldots, \ldots, \ldots$,

Joint Program in Oceanographic Engineering Massachusetts Institute of Technology , Woods Hole Oceanographic Institution

Certified by

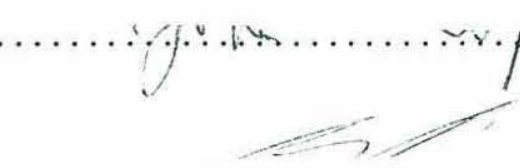

Certified by

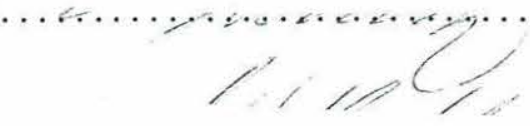

Accepted by

\section{Woods}

Dr. John L. SpiesbergerL IBRARY LABORATORY 


\title{
Basin-Scale Tidal Measurements using Acoustic Tomography \\ by
}

\author{
Robert Hugh Headrick
}

\author{
Submitted to the Massachusetts Institute of Technology/ \\ Woods Hole Oceanographic Institution \\ Joint Program in Oceanographic Engineering \\ on August 10, 1990, in partial fulfillment of the \\ requirements for the degrees of \\ Ocean Engineer \\ and \\ Master of Science in Ocean Engineering
}

\begin{abstract}
Travel-times of acoustic signals were measured between a bottom-mounted source near Oahu and four bottom-mounted receivers located near Washington, Oregon, and California in 1988 and 1989. This paper discusses the observed tidal signals. At three out of four receivers, observed travel times at $M 2$ and $S 2$ periods agree with predictions from barotropic tide models to within $\pm 30^{\circ}$ in phase and a factor of 1.6 in amplitude. The discrepancy at the fourth receiver can be removed by including predicted effects of phase-locked baroclinic tides generated by seamounts.

Our estimates of barotropic $M 2$ tidal dissipation by seamounts vary between $2 \times 10^{16}$ and $1 \times 10^{18} \mathrm{erg} \cdot \mathrm{s}^{-1}$. The variation by two orders of magnitude is due to uncertainties in the numbers and sizes of seamounts. The larger dissipation $\left(1 \times 10^{18} \mathrm{erg} \cdot \mathrm{s}^{-1}\right)$ is the same order as previous estimates and amounts to $4 \%$ of the total dissipation at $M 2$.
\end{abstract}

Thesis Supervisor: Dr. John L. Spiesberger

Woods Hole Oceanographic Institution 


\section{Acknowledgments}

I owe many thanks to my thesis advisor, Dr. John Spiesberger, for his guidance. His knowledge, understanding, and insight enabled the completion of this thesis.

I thank a fellow Naval Officer, Paul Bushong. He laid the foundations for much of this work while studying at MIT/WHOI from 1985 to 1987.

I am indebted to the United States Navy for giving me the opportunity to pursue my graduate studies at MIT and WHOI.

I thank Carolyn, my wife, for her enduring love and support.

Finally, I thank the Lord Jesus Christ, without whom I could do nothing. 


\section{Contents}

1 Introduction $\quad 8$

2 Data Acquisition $\quad 11$

3 Data Analysis $\quad 13$

3.1 Barotropic Model for Acoustic travel-time . . . . . . . . . . . . . . 13

3.2 Barotropic Model Comparisons with Observed Tidal Signals . . . . . . . . 14

3.2 .1 Tidal Signal Estimation . . . . . . . . . . . . . . . 14

3.2 .2 Comparison with Model . . . . . . . . . . . . . 15

3.2 .3 Analysis ........................... 16

3.3 Baroclinic Tide Prediction . . . . . . . . . . . . . . 18

$3.3 .1 \quad$ Phase-Stable Internal Tides . . . . . . . . . . . . . . . . . 18

3.3.2 Influence on Travel-Time at Receiver R4 . . . . . . . . . . . . . 19

4 Tidal Dissipation $\quad 21$

4.1 Tidal Dissipation at $M 2$ from Satellite Observations . . . . . . . . . . 21

4.2 Tidal Dissipation by Seamounts . . . . . . . . . . . . . . 22

5 Conclusions $\quad 25$

$\begin{array}{ll}\text { A Obtaining Tidal Currents from Elevations } & 27\end{array}$

B Guyot-Generated Internal Tides $\quad 33$

$\begin{array}{ll}\text { C Baroclinic Tide Travel-Time Model } & 39\end{array}$ 
$\begin{array}{ll}\text { D Signal Processing } & 41\end{array}$

D.1 The Transmitted Signal . . . . . . . . . . . . . . . . 41

D.2 Post-Detection Processing . . . . . . . . . . . . . . 42

D.2.1 Demodulation and Averaging . . . . . . . . . . 42

D.2.2 Replica Correlation . . . . . . . . . . . . . . . . 43

D.2.3 Phase Change Between Sequence Records . . . . . . . . . . . . 43 


\section{List of Figures}

$1-1 \quad$ Source and Receivers . . . . . . . . . . . . . . 9

2-1 Available Tomography Data . . . . . . . . . . . . . . . . 12

3-1 Phase-Difference Power-Spectra. . . . . . . . . . . . . . 15

3-2 Data to Barotropic Model Comparisons. . . . . . . . . . . . 17

3-3 R4 Data-to-Model Comparisons . . . . . . . . . . . . . 20

A-1 M2 Currents in the NE Pacific . . . . . . . . . . . . 30

A-2 $K 1$ Currents in the NE Pacific. . . . . . . . . . . . . . 32

B-1 Two-Layer Ocean Model . . . . . . . . . . . . . . . . 34

B-2 R4 Prediction Error as a Function of Reduced Gravity . . . . . . . . 38 


\section{List of Tables}

3.1 Barotropic Model Signal Amplitudes. . . . . . . . . . . . . . . . . 16

$3.2 M 2$ and $S 2$ Internal Tide Corrected Travel-Times at Receiver R4. . . . 19

$3.3 N 2$ and $K 2$ Internal Tide Corrected Travel-Times at Receiver R4. . . . . 20

4.1 Seamount Distribution Models. . . . . . . . . . . . . . . . . . 23

4.2 Seamount Dimensions. . . . . . . . . . . . . . . . . 23

A.1 Major Tidal Frequencies . . . . . . . . . . . . . . . . 28

B.1 Generalized Two-layer Internal Tide Solution. . . . . . . . . . . . 36

B.2 Paticular Two-layer $M 2$ and $S 2$ Internal Tide Solutions. . . . . . . . . 37 


\section{Chapter 1}

\section{Introduction}

We measure travel-times of acoustic signals between a bottom-mounted source near Oahu and four bottom-mounted receivers located near Washington, Oregon, and California in 1988 and 1989 (Figure 1-1). The power-spectra of basin-scale travel-times exhibit prominent variability at tidal periods [Spiesberger et al., 1989b]. There are two important reasons for further study of the tidal signals. First, numerical models for barotropic tides can be validated by using these new observations. Second, it is important to detide tomography data to better study other ocean fluctuations at similar periods [Spiesberger et al., 1989b].

The tides can be separated into barotropic and baroclinic modes, and their associated influences on travel-time can be separated into current and displacement effects. The tidal currents affect travel-time by alternating between alliance and opposition to the direction of sound propagation. The rise and fall of the water column affects the travel-time by causing periodic vertical iso-therm displacements and pressure fluctuations. Worcester et al. [1990] has shown that barotropic current effects can be quantified by using reciprocal transmissions; one-way transmissions are used in this experiment, so we quantify barotropic effects by comparing measured data to barotropic tide model predictions.

We find that acoustic travel-times computed from barotropic tidal models are similar to observations at three out of four receivers. The discrepancy at the fourth receiver can be understood by including the effects of phase-locked baroclinic tides generated by seamounts near the transmission path. Generation mechanisms are important both for 


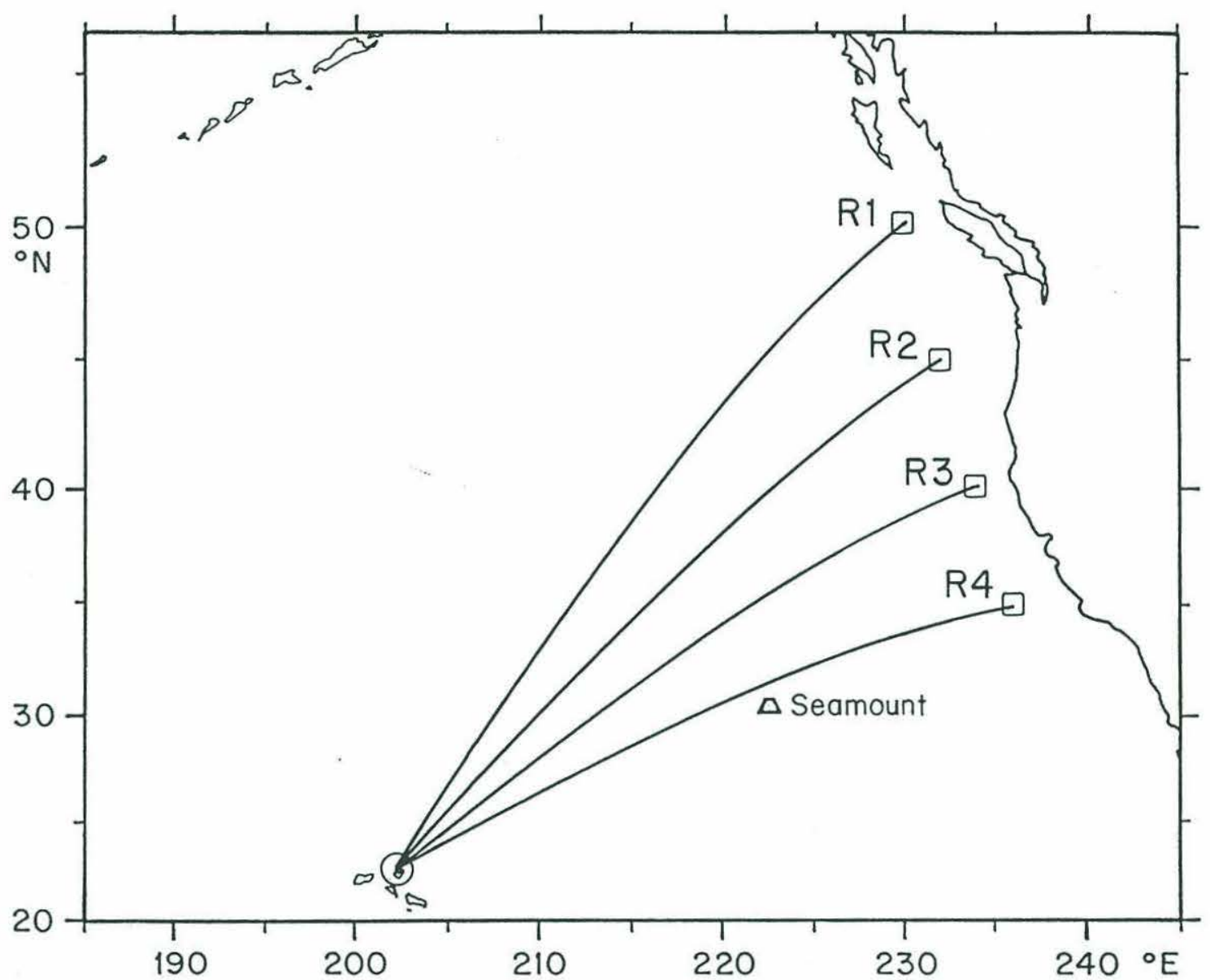

Figure 1-1: The acoustic tomography experiments of 1988 and 1989 consist of a source located near Kaneohe Bay, Oahu, and four bottom receivers (R1 to R4) whose approximate positions are shown. The distances between source and receivers are all about $4000 \mathrm{~km}$. 
understanding the distribution of internal wave energy and the energy budget of tidal dissipation [Munk, 1966; Wunsch, 1975; Bell, 1975; Lambeck, 1980; Hendershott, 1981].

This report closely follows Headrick et al. [1990] and is organized as follows. Chapter 2 discusses the experiment and the estimation of acoustic travel-time. Comparisons between observations and predictions from barotropic and baroclinic tide models are made in Chapter 3. Chapter 4 predicts the global dissipation of tidal energy at $M 2$ due to seamounts. Conclusions follow in Chapter 5. 


\section{Chapter 2}

\section{Data Acquisition}

The travel-time differences of pulse-like signals are measured at 3 minute intervals in 1988 and 2 minute intervals in 1989 between the source at Oahu and the four receivers (Figure 1-1). Travel-times are intermittently measured for a total of between four and eight months (Figure 2-1). The transmission distances are about $4000 \mathrm{~km}$ and the transit times are about 40 minutes.

Spiesberger et al. [1989a,b] describes the maximum likelihood estimator of phase differences used to estimate differences in travel-time. The basic process is outlined in Appendix D. With this estimator, the change in acoustic phase at $133 \mathrm{~Hz}, \delta \phi$, is related to the travel-time difference, $\delta \tau$, by,

$$
\delta \tau=\frac{2 \pi \delta \phi}{\omega_{c} \omega \Delta t}
$$

where $\omega_{c}=2 \pi \cdot 133\left(\mathrm{rad} \cdot \mathrm{s}^{-1}\right)$ is the center radian frequency of the transmitted signal, $\omega$ is the radian frequency of the travel-time oscillation, and $\Delta t$ is the geophysical time interval over which travel-time differences are measured (184 s in 1988, $122 \mathrm{~s}$ in 1989) [Spiesberger et al., 1989a]. 


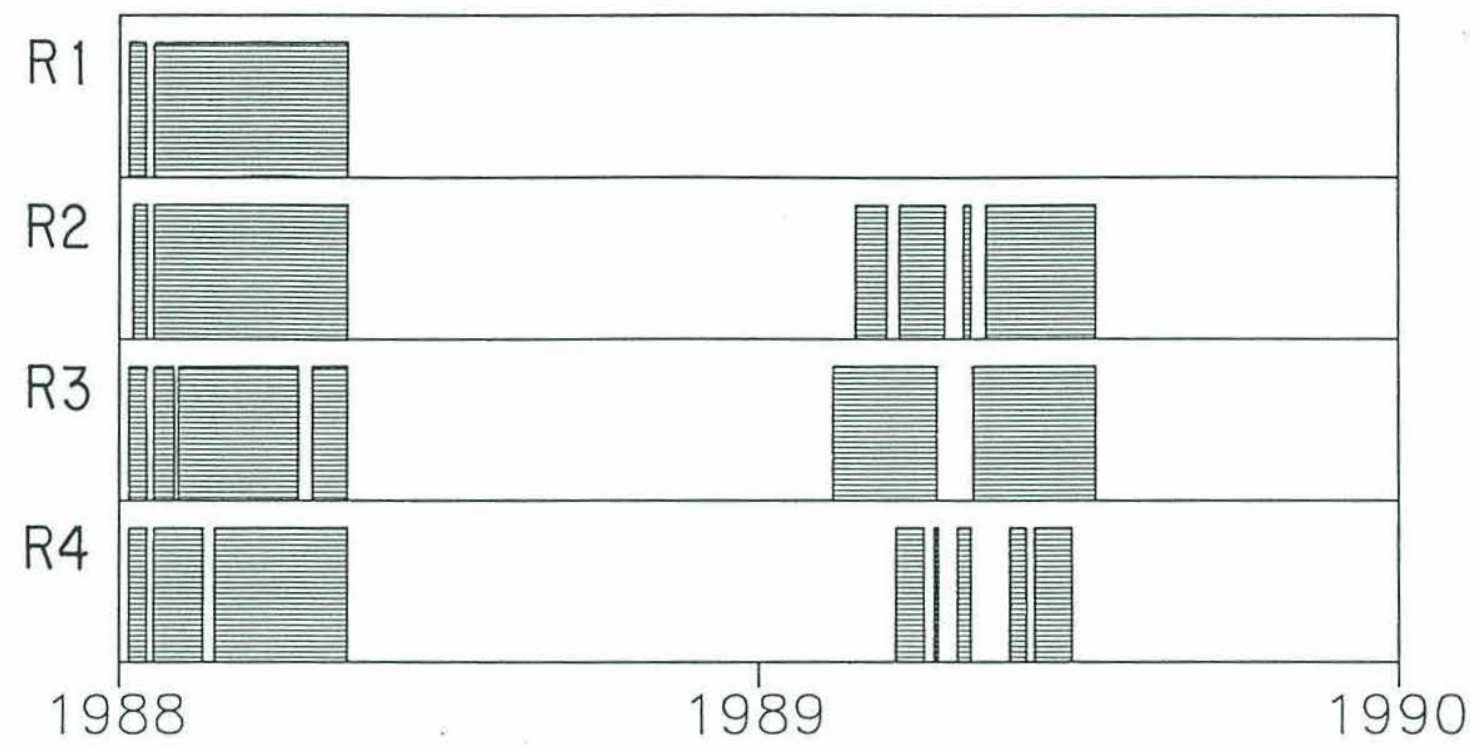

Figure 2-1: Shaded regions indicate the times when tomographic data were recorded at each of the receivers. 


\section{Chapter 3}

\section{Data Analysis}

\subsection{Barotropic Model for Acoustic travel-time}

The change in acoustic travel-time due to barotropic tides is given by,

$$
\Delta T_{\mathrm{bt}}\left(t, \Gamma_{\mathrm{R}}\right)=\frac{-1}{c_{0}^{2}}\left[\int_{\Gamma_{\mathrm{R}}} \vec{u}\left(\vec{s}, t-\frac{l(\vec{s})}{c_{o}}\right) \cdot d \vec{s}+k_{1} \int_{\Gamma_{\mathrm{R}}} \zeta\left(\vec{s}, t-\frac{l(\vec{s})}{c_{o}}\right) d s\right],
$$

where the geophysical time at the receiver is $t$, the geodesic between the source and receiver is $\Gamma_{\mathrm{R}}$, and the mean sound speed is $c_{o}$. The barotropic current and surface displacement at location $\vec{s}$ on the geodesic at time $t$ are denoted by $\vec{u}\left(\vec{s}, t-\frac{l(\vec{s})}{c_{o}}\right)$ and $\zeta\left(\vec{s}, t-\frac{l(\vec{s})}{c_{o}}\right)$ respectively. The incremental on the geodesic is $d s$, and $l(\vec{s})$ is the distance from the receiver. The constant is $k_{1}=3.9 \times 10^{-3}$ [Spiesberger et al., 1989b]. The effects of finite propagation time are taken into account by the correction term $\left(-\frac{l(\vec{s})}{c_{o}}\right)$. The finite propagation delay amounts to a phase correction of order $\pm 10^{\circ}$ at semidiurnal periods.

The first term in (3.1) contains the effect of propagating sound with and against the tidal current. The second term, which amounts to a 1 or $2 \mathrm{~ms}$ correction, contains the combined effects of vertical iso-therm displacement and pressure fluctuations which accompany the rise and fall of the sea surface. For barotropic tides the travel-time change is about the same for different acoustic multipaths [Spiesberger et al., 1989a].

The elevation, $\zeta$, is obtained from the Naval Surface Weapons Center (NSWC) tide model derived by Schwiderski [1978]. Unfortunately, except for M2, NSWC models of the tidal current field are unavailable. We therefore use a linearized version of Schwiderski's 
[1978] Laplace tidal equations (LTE) to predict tidal currents. The equations are,

$$
\begin{aligned}
& \frac{\partial u}{\partial t}=2 \Omega \cos \bar{\theta} v+\frac{g}{R_{e} \sin \bar{\theta}}\left[\frac{\partial}{\partial \lambda}(\alpha \eta-\beta \zeta)\right]-k_{d} u, \\
& \frac{\partial v}{\partial t}=-2 \Omega \cos \bar{\theta} u-\frac{g}{R_{e}}\left[\frac{\partial}{\partial \bar{\theta}}(\alpha \eta-\beta \zeta)\right]-k_{d} v,
\end{aligned}
$$

where $\lambda$ and $\bar{\theta}$ are longitude and colatitude respectively, $u$ and $v$ are east and north current speeds respectively, $\eta$ is Newton's equilibrium tide (which is proportional to the astronomical tide generating potential), $g$ is the earth's gravitational attraction, $R_{e}$ is the radius of a spherical earth, $\Omega$ is the Earth's angular rate of rotation, and $k_{d}$ is a damping coefficient. The constants $\alpha$ and $\beta$ derived by Schwiderski [1978] correct for the solid-earth tide, ocean tidal loading of the solid earth, and the associated gravity potential perturbations of all tides and loadings. The solution to (3.2) for $u$ and $v$ is outlined in Appendix A.

Equation (3.1) is decomposed into contributions from each tidal frequency, $\omega_{k}$, of interest, and solutions are determined by collecting and integrating $\sin \omega_{k} t$ and $\cos \omega_{k} t$ terms separately. The resulting travel-time change model is,

$$
\Delta T_{\mathrm{bt}}\left(t, \Gamma_{\mathrm{R}}\right)=\sum_{k=1}^{K} \mathrm{e}_{k}\left(\Gamma_{\mathrm{R}}\right) \cos \omega_{k} t+\mathrm{f}_{k}\left(\Gamma_{\mathrm{R}}\right) \sin \omega_{k} t,
$$

where $e_{k}\left(\Gamma_{\mathrm{R}}\right)$ and $\mathrm{f}_{k}\left(\Gamma_{\mathrm{R}}\right)$ are the solutions to the separated integrals, and $K$ is the total number of tidal frequencies calculated.

\subsection{Barotropic Model Comparisons with Observed Tidal Signals}

\subsubsection{Tidal Signal Estimation}

Power-spectra of the differences in acoustic-phase exhibit prominent variability at tidal periods [Spiesberger et al., 1989a,b] (Figure 3-1). For each receiver, tidal signals are estimated by fitting the 8 most prominent tidal components to the data (Appendix A, Table A.1). Specifically, we determine $\mathrm{a}_{k}$ and $\mathrm{b}_{k} ; k=1,2, \ldots, 8$, in,

$$
\delta \phi\left(t_{n}, \Gamma_{\mathrm{R}}\right)=\sum_{k=1}^{8} \mathrm{a}_{k}\left(\Gamma_{\mathrm{R}}\right) \cos \omega_{k} t_{n}+\mathrm{b}_{k}\left(\Gamma_{\mathrm{R}}\right) \sin \omega_{k} t_{n} ; n=1,2, \ldots, N,
$$




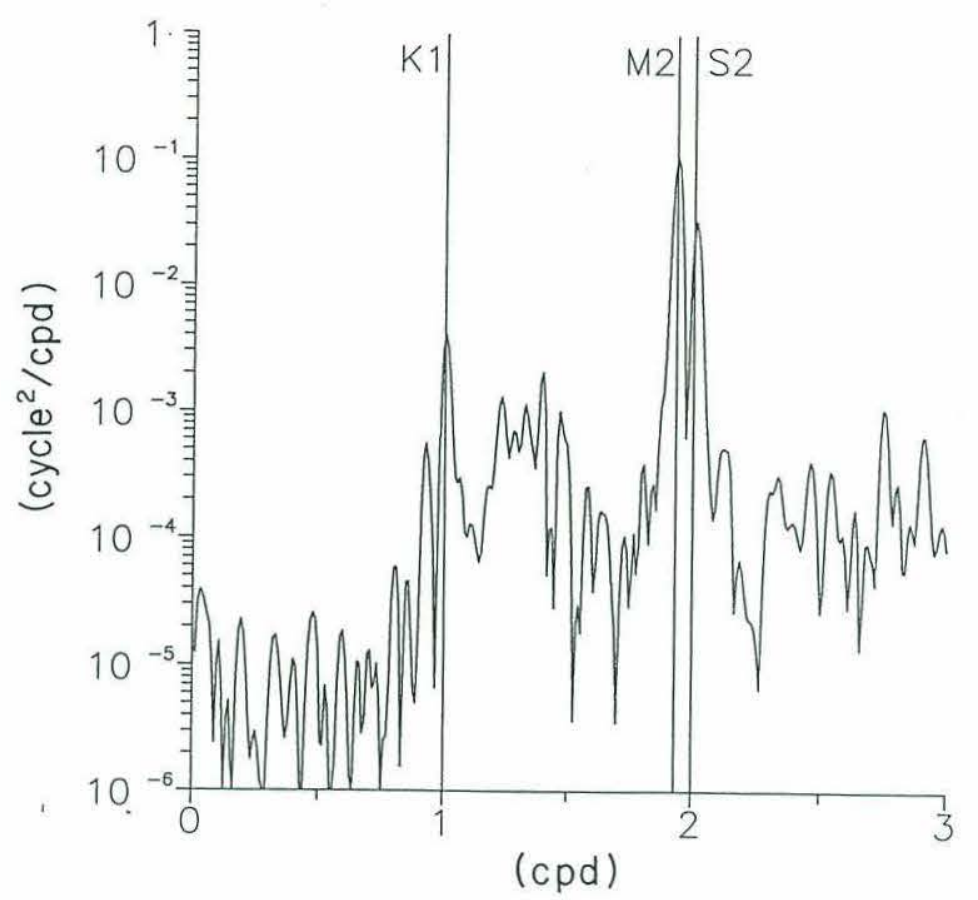

Figure 3-1: Power spectral density estimate for the $133 \mathrm{~Hz}$ phase-differences at receiver R2. This periodogram uses a Bartlett data window with 42 days of data obtained in 1988 . The tidal peaks at the $K 1, M 2$, and $S 2$ frequencies have rms values which correspond to travel-time change amplitudes of $8.6,23.5$, and 12.3 milliseconds respectively.

by linear least squares [Lawson and Hanson, 1974], where the phase-differences are measured at times $t_{n}$, and the total number of measurements equals $N$. Depending on the receiver, $N$ equals 55,000 to 135,000 , so the least squares problem is overdetermined. Each least-squares fit to phase-differences is compared to barotropic model travel-times to determine how much of the signal can be attributed to barotropic tidal effects.

\subsubsection{Comparison with Model}

Assuming all of the (3.4) signal is due to barotropic influences, each tidal component is proportional to the time derivative of the corresponding (3.3) model. Comparisons between observations and the barotropic model are illustrated by the phasors of Figure 3-2. Each phasor magnitude corresponds to a data-to-model signal amplitude ratio, which 


\begin{tabular}{ccccc}
\hline \hline Frequency & $\begin{array}{c}\mathrm{R} 1 \\
\mathrm{Amp}(\mathrm{ms})\end{array}$ & $\begin{array}{c}\mathrm{R} 2 \\
\mathrm{Amp}(\mathrm{ms})\end{array}$ & $\begin{array}{c}\mathrm{R} 3 \\
\mathrm{Amp}(\mathrm{ms})\end{array}$ & $\begin{array}{c}\mathrm{R} 4 \\
\mathrm{Amp}(\mathrm{ms})\end{array}$ \\
\hline M2 & 26 & 22 & 15 & 7 \\
$S 2$ & 11 & 7 & 4 & 3 \\
$K 1$ & 10 & 7 & 1 & 3 \\
\hline
\end{tabular}

Table 3.1: Predicted acoustic signal amplitudes for barotropic tides. The model amplitudes, $\left(\mathrm{e}_{k}^{2}+\mathrm{f}_{k}^{2}\right)^{1 / 2}$, are determined from the constants in (3.3) and have rms errors of about $2 \mathrm{~ms}$.

from (2.1) is,

$$
\frac{2 \pi\left(\mathrm{a}_{k}^{2}+\mathrm{b}_{k}^{2}\right)^{1 / 2}}{\omega_{c} \omega_{k} \Delta t\left(\mathrm{e}_{k}^{2}+\mathrm{f}_{k}^{2}\right)^{1 / 2}},
$$

and each phasor angle corresponds to a difference between data and model signal phases, which is given by,

$$
\tan ^{-1}\left(\frac{\mathrm{a}_{k}}{\mathrm{~b}_{k}}\right)-\tan ^{-1}\left(\frac{\mathrm{e}_{k}}{\mathrm{f}_{k}}\right)+\frac{\pi}{2}
$$

\subsubsection{Analysis}

First, we discuss the comparison at two semidiurnal periods ( $M 2$ and $S 2$ ). Predicted tidal amplitudes vary from 3 to $26 \mathrm{~ms}$ at our receivers (Table 3.1). At receivers R1, R2, and R3, observed amplitudes are 0.8 to 1.6 times predicted amplitudes (Figure 3-2). At R4 the observed amplitudes are 2.4 and 3.4 times predictions. Predicted and observed phases are within $\pm 30^{\circ}$ at R1, R2, and R3 but differ by up to $47^{\circ}$ at R4. Data at R1, R2, and R3 agree with predictions from the barotropic model to first order, but at R4, the barotropic model is inadequate. We return to this problem in the next section.

We next discuss the comparisons for one semidiurnal period (K1). The models for barotropic currents at diurnal periods are inaccurate within a $\pm 10^{\circ}$ band centered at $30^{\circ}$ $\mathrm{N}$ (Appendix A). The transmissions to all receivers intersect this band, so comparisons between predictions and observations are problematic. Nonetheless, predictions at R1 


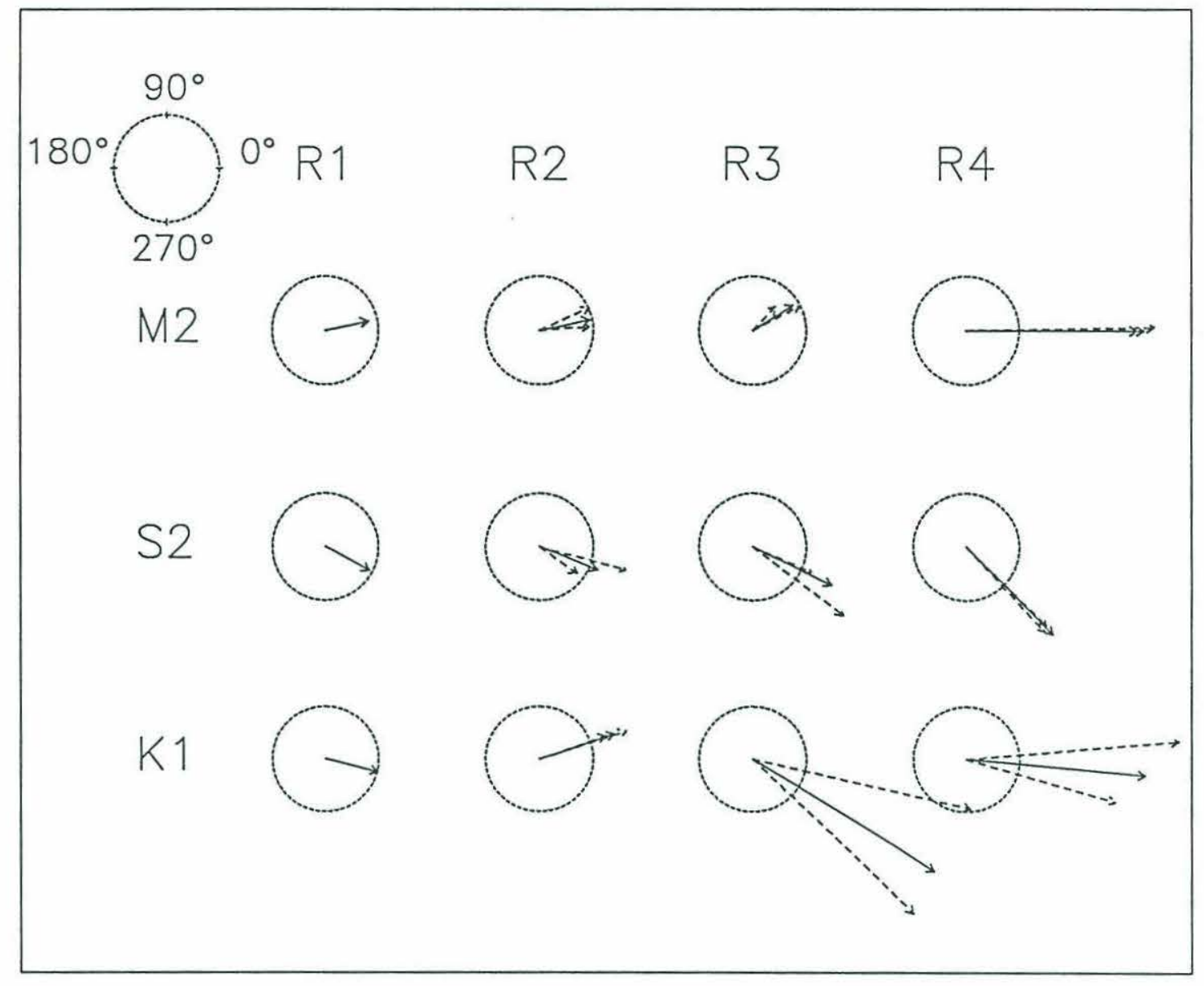

Figure 3-2: Comparison of phase and amplitude of measured and predicted tidal signals at four receivers (R1 to R4) and three frequencies $(M 2, S 2, K 1)$. Predicted tidal signals are obtained from the barotropic model given by (3.1). Each vector angle corresponds to a measurement phase that leads the predicted phase by the indicated amount. The unit circle in the upper left hand corner illustrates the vector angle convention. Vector magnitude corresponds to the ratio of measured amplitude to predicted amplitude. Predicted magnitudes are listed in Table 3.1. An exact match between measurement and prediction is represented by a vector of angle zero and length one (tip just touching the unit circle at zero degrees). Solid arrows correspond to measurements of the combined 1988 and 1989 record, and the dashed arrows correspond to separate 1988 and 1989 data measurements. 
and $\mathrm{R} 2$ resemble observations at tidal period $\mathrm{K} 1$ (Figure 3-2). It may be that R1 and R2 tidal signals are similar to predictions because their transmission paths travel smaller distances within $30^{\circ} \pm 10^{\circ} \mathrm{N}$ than paths to R3 and R4 (Figure 1).

\subsection{Baroclinic Tide Prediction}

\subsubsection{Phase-Stable Internal Tides}

Apart from generation regions, baroclinic tides are not phase-locked to the ATGF, and their phases are incoherent at $100 \mathrm{~km}$ distance (approximately an internal tide wavelength) [Hendershott,1981]. In generation regions, baroclinic tides may be phase-locked to the ATGF [Munk et al., 1981]. Open ocean evidence of this phase-lock is seen in a phasestable $M 2$ baroclinic tide over a large guyot east of Hawaii [Noble et al., 1988]. The transmission path to R4 passes within a few hundred kilometers of several large seamounts, one of which is indicated in Figure 1-1. We hypothesize that the semidiurnal signals at R4 do not agree with predictions from the barotropic model because of the presence of phase-locked baroclinic tides near the seamounts.

The transmission paths to R1, R2, and R3 may be near other large seamounts, but because there are no large discrepancies between the data and barotropic model predictions, it is unlikely that internal tides play a zero order role in determining tidal signals at these receivers.

Appendix B summarizes a model for the generation of baroclinic tides from the interaction between barotropic currents and seamounts. For baroclinic tides, acoustic traveltimes are modulated by sound-speed fluctuations associated with vertical straining of iso-tachs [Spiesberger et al., 1989b]. The effect for the $j$ th acoustic multipath, $\Gamma_{\mathrm{R}}(j)$, is,

$$
\Delta T_{\mathrm{bc}}\left(t, \Gamma_{\mathrm{R}}(j)\right)=\frac{1}{c_{0}^{2}} \int_{\Gamma_{\mathrm{R}}(j)} \zeta_{\text {int }}\left(\vec{r}, t-\frac{l(\vec{r})}{c_{o}}\right) \frac{\partial c_{p}(\vec{r})}{\partial z} d r
$$

where the position along the multipath is $\vec{r}$, the vertical displacement of the internal tide

is $\zeta_{\text {int }}$, and the vertical derivative of the potential sound-speed is $\frac{\partial c_{p}(\vec{r})}{\partial z}$. 


\begin{tabular}{|c|c|c|c|c|}
\hline & \multicolumn{2}{|l|}{$M 2$} & \multicolumn{2}{|l|}{$S 2$} \\
\hline & Amplitude(ms) & $\operatorname{Phase}\left({ }^{\circ}\right)$ & Amplitude(ms) & $\operatorname{Phase}\left({ }^{\circ}\right)$ \\
\hline Barotropic Model & 7 & 113 & 3 & 324 \\
\hline Baroclinic Model & 20 & 117 & 5 & 265 \\
\hline Combined Model & 27 & 116 & 7 & 286 \\
\hline Measured Data & $24 \pm 1$ & $114 \pm 1$ & $7 \pm 0.1$ & $279 \pm 1$ \\
\hline
\end{tabular}

Table 3.2: Comparison of predicted and observed travel-time changes at $M 2$ and $S 2$ for receiver R4. The uncertainties on the measured data are based on the differences between 1988 and 1989 least-square fits. Phases are based on a $\sin \omega t$ time dependence with $t=0$ at 0000 Greenwich mean time on 1 January 1989.

\subsubsection{Influence on Travel-Time at Receiver R4}

Being the southern-most receiver, R4 has a more east-west oriented ray path than the other receivers. This orientation results in a weaker influence from the generally north-south oriented $M 2$ and $S 2$ barotropic tidal currents, so the effects of phase-locked baroclinic tides are more apparent. The predicted $M 2$ baroclinic tide, generated from one of several seamounts near the transmission path to R4 (Figure 1-1), has an amplitude and wavelength of $6.2 \mathrm{~m}$ and $98 \mathrm{~km}$ respectively (Appendix B) and produces a $21 \mathrm{~ms}$ amplitude travel-time signal (Appendix C).

The sum of the predicted $M 2$ baroclinic tidal signals, generated from all prominent seamounts along the transmission path, is added to the barotropic prediction. The barotropic plus baroclinic prediction for $\mathrm{R} 4$ is almost identical to observations at $M 2$ and $S 2$ (Figure 3-3 and Table 3.2). The prediction is improved at $K 2$ but not at $N 2$ (Table 3.3). The frequency at $N 2$ is close to $M 2$ (Table A.1), and the observed amplitude at $N 2$ is small. We suspect that the barotropic plus baroclinic prediction does not fit observations at $N 2$, because strong baroclinic tides at $M 2$ may be smeared into $N 2$ by dynamic processes [Hendershott, 1981]. 


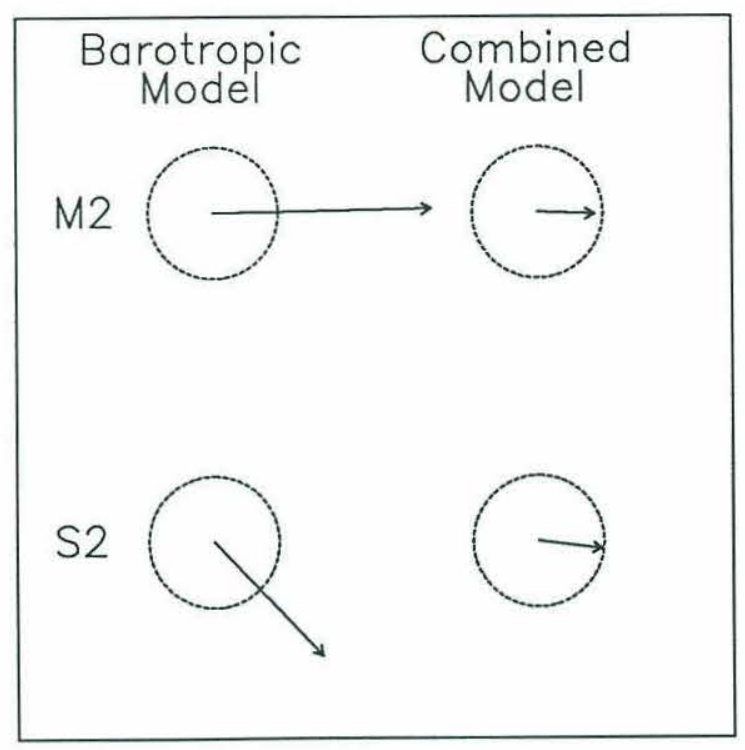

Figure 3-3: The phasors on the left are identical to the combined 1988 and 1989 R4 record phasors shown in Figure 3-2. The phasors on the right present the same analysis using the combined barotropic and baroclinic models in Table 3.2 .

\begin{tabular}{|c|c|c|c|c|}
\hline \multirow[b]{3}{*}{ Barotropic Model } & \multicolumn{2}{|l|}{$N 2$} & \multicolumn{2}{|l|}{$K 2$} \\
\hline & Amplitude(ms) & Phase $\left(^{\circ}\right)$ & Amplitude(ms) & $\operatorname{Phase}\left({ }^{\circ}\right)$ \\
\hline & 1 & 37 & 1 & 153 \\
\hline Baroclinic Model & 6 & 315 & 3 & 117 \\
\hline Combined Model & 6 & 325 & 4 & 126 \\
\hline Measured Data & $2 \pm 1$ & $331 \pm 38$ & $5 \pm 2$ & $153 \pm 18$ \\
\hline
\end{tabular}

Table 3.3: Same as Table 3.2, except comparisons are at $N 2$ and $K 2$ frequencies. 


\section{Chapter 4}

\section{Tidal Dissipation}

The effect of the ocean tidal bulge on the Earth-Moon system is a well known phenomena that is discussed extensively by Lambeck [1980]. The friction-induced phase lag between $\zeta$ and $\eta$ produces a torque in the Earth-Moon system that is responsible for the lengthening of the day and recession of the Moon at rates of about $1.8 \mathrm{~ms} \cdot \operatorname{century}^{-1}$ and 3.7 $\mathrm{m} \cdot$ century $^{-1}$ respectively [Christodoulidis, et al. 1988]. The applied torque transfers an amount of orbital momentum to the Moon equal to the rotational momentum lost by the Earth [Lambeck, 1977]. The net result of this momentum transfer is an overall reduction in system kinetic and potential energy. System energy is dissipated for the most part in oceanic tides, with some fraction (which has yet to be quantified) occurring through conversion to baroclinic tides [Lambeck, 1980]. In this chapter we estimate the barotropicto-baroclinic conversion rate of the $M 2$ tide at seamounts.

\subsection{Tidal Dissipation at $M 2$ from Satellite Observations}

The rate of energy dissipation is,

$$
\frac{d E}{d t}=\left(5.86 \frac{d \Omega_{T}}{d t}-9.58 \frac{d n}{d t}\right) 10^{40} \mathrm{erg} \cdot \mathrm{s}^{-1}
$$

where $\frac{d \Omega_{T}}{d t}$ is the total tidal acceleration of the Earth and $\frac{d n}{d t}$ is the acceleration of the Moon's orbit in $\mathrm{rad} \cdot \mathrm{s}^{-2}[$ Lambeck, 1977]. Recent estimates for $M 2$ accelerations have 
been obtained from the orbital perturbations of 17 satellites, and have values given by,

$$
\begin{aligned}
& \frac{d \Omega_{T}}{d t}=-4.45 \times 10^{-22} \mathrm{rad} \cdot \mathrm{s}^{-2}, \\
& \frac{d n}{d t}=-9.73 \times 10^{-24} \mathrm{rad} \cdot \mathrm{s}^{-2},
\end{aligned}
$$

[Christodoulidis, et al., 1988]. Substituting (4.2) into (4.1) yields $2.5 \times 10^{19} \mathrm{erg} \cdot \mathrm{s}^{-1}$ at $M 2$ (about $75 \%$ of all tidal dissipation).

\subsection{Tidal Dissipation by Seamounts}

We consider two models for the distributions of seamounts. In the first model the number of seamounts with a basal radius $r \geq R(\mathrm{~km})$ is given by,

$$
N(R)=1.44 \times 10^{6} \exp [-0.63 R]
$$

Equation (4.3) is an extrapolation of Jordan et al.'s [1983] results, for the eastern Pacific, to the world ocean. In the second model, the number of seamounts is estimated from altimeter measurements by Seasat [Craig and Sandwell, 1988]. Seasat detects many large seamounts which are absent in the model by Jordan et al. [1983], but it does not detect small seamounts, as the altimeter profile sensitivity to seamounts falls off rapidly below a basal radius of $20 \mathrm{~km}$ [Craig et al., 1988]. These two seamount distributions are outlined in Table 4.1.

The rate of energy conversion from barotropic to baroclinic tides at each seamount is approximately,

$$
\frac{d E}{d t}=2(\Delta x) c_{g i} \overline{E_{i}},
$$

where $\Delta x$ is the horizontal dimension of each seamount perpendicular to the direction of barotropic current, $c_{g i}$ is the group speed of the internal tide (Equation (B.22)), and $\overline{E_{i}}$ is the average energy density of the internal tide (Equation (B.21)). The dimension, $\Delta x$, is obtained from the seamount's basal radius according to Table 4.2. If we take the barotropic

current speed to equal $2 \mathrm{~cm} \cdot \mathrm{s}^{-1}$ and the inertial frequency to equal $0.73 \times 10^{-4} \mathrm{~s}^{-1}\left(30^{\circ}\right.$ $\mathrm{N})$, then equations (4.4) and (B.23) yield a total energy conversion rate of $2 \times 10^{16}$ and $1 \times 10^{18} \mathrm{erg} \cdot \mathrm{s}^{-1}$ for the exponential and Seasat distributions respectively. These estimates are proportional to $\Delta x$ and the squares of both seamount height and the barotropic 


\begin{tabular}{|c|c|c|}
\hline \multirow{3}{*}{$\begin{array}{c}\text { Basal Radius } \\
\mathrm{r}(\mathrm{km})\end{array}$} & \multicolumn{2}{|c|}{ Number of Seamounts } \\
\hline & Exponential & Seasat \\
\hline & Model & Model \\
\hline $1-2$ & 360,000 & 0 \\
\hline $2-4$ & 290,000 & 0 \\
\hline $4-6$ & 83,000 & 0 \\
\hline $6-10$ & 30,000 & 200 \\
\hline $10-15$ & 2500 & 1200 \\
\hline $15-20$ & 110 & 6000 \\
\hline $20-26$ & 5 & 8800 \\
\hline $26-32$ & 0 & 6800 \\
\hline $32-38$ & 0 & 4800 \\
\hline $38-44$ & 0 & 2800 \\
\hline $44-50$ & 0 & 1600 \\
\hline
\end{tabular}

Table 4.1: Seamount distribution for the world's oceans as a function of basal radius by Jordan et al. [1983] (exponential model) and Craig and Sandwell [1988] (Seasat model). The Seasat model is obtained by converting Craig and Sandwell's [1988] histogram of peak-to-trough separation, $\mathrm{p}$, to basal radius using $r=(0.83) \mathrm{p}$ in accordance with measured seamount slopes. The numbers so obtained are multiplied by four to account for undetected seamounts [Craig et al., 1988]. Some of the discrepancy between models may be due to large seamounts in the western Pacific that were not represented in the Jordan et al. [1983] survey.

\begin{tabular}{|c|c|c|}
\hline \multicolumn{3}{|c|}{ Rectangular Seamount Dimensions } \\
\hline & Exponential & Seasat \\
\hline Dimension & Model & Model \\
\hline$\Delta x$ & $(0.62) r$ & $(0.62) r$ \\
\hline$\Delta z$ & $(0.21) r$ & $2500 \mathrm{~m}$ \\
\hline
\end{tabular}

Table 4.2: Seamount dimensions for the exponential and Seasat models (Table 4.1). The length and width of each seamount is $\Delta x$, and the height is $\Delta z$. Basal radius is denoted by $r$. The scale factor for $\Delta x,(0.62)$, is derived from a mean flatness of 0.31 reported for 85 seamounts in the Pacific [Smith, 1988]. The $\Delta z$ scale factor for the exponential model is derived from the mean height-to-basal-radius ratio of 0.21 [Smith, 1988]. The height is set to $2500 \mathrm{~m}$ for the Seasat model. 
current speed (Equation (B.23)). The exponential model yields insignificant dissipation because it contains relatively few wide seamounts (Table 4.1). The Seasat model yields a dissipation that is about $4 \%$ of the total at M2. Munk [1966] and Bell [1975] obtained rates of $5.0 \times 10^{18}$ and $2.5 \times 10^{18} \mathrm{erg} \cdot \mathrm{s}^{-1}$ respectively for the conversion to baroclinic tides by bottom roughness. These estimates are the same order as we obtain using the Seasat distribution. 


\section{Chapter 5}

\section{Conclusions}

We reach the following conclusions:

1. Because barotropic signal predictions at R1, R2, and R3 compare favorably with observations and because barotropic wave-lengths have basin-scales, the principle discrepancy between barotropic signal predictions and observations at R4 is probably not due to errors in Schwiderski's [1978] model. Furthermore, because barotropic plus baroclinic signal predictions compare favorably with observations at R4, we believe the Schwiderski [1978] model is adequate for barotropic predictions.

2. Seamounts may be responsible for a small ( $\sim 5 \%$ ), but non-negligible, fraction of the dissipation of barotropic tides. The dissipation is sensitive to the heights, radii, and numbers of seamounts (all poorly known).

3. The generation of baroclinic tides at seamounts can be accounted for with a twolayer model of the ocean (Appendix B).

4. Barotropic tidal currents can be predicted from Schwiderski's [1978] tidal elevation predictions with our model (Appendix A), except within $\pm 10^{\circ}$ of $30^{\circ}$ North and South (diurnal frequencies) and near $90^{\circ} \mathrm{N}$ (semidiurnal frequencies).

5. Comparison of measured tidal signals (from basin-scale acoustic transmissions) with predicted tidal signals (from barotropic models) can be used to detect baroclinic tides which are phase-locked to the ATGF. 
6. It is difficult to make a-priori predictions of tidal signals in basin-scale transmissions, because generation regions of baroclinic tides are poorly mapped. 


\section{Appendix A}

\section{Obtaining Tidal Currents from}

\section{Elevations}

We solve (3.2) for $u$ and $v$ by defining,

$$
\begin{aligned}
& u=b_{1} \cos \omega t+b_{2} \sin \omega t, \\
& v=a_{1} \cos \omega t+a_{2} \sin \omega t,
\end{aligned}
$$

and,

$$
\begin{aligned}
& \frac{g}{R \sin \bar{\theta}}\left[\frac{\partial}{\partial \lambda}(\alpha \eta-\beta \zeta)\right]=y_{1} \cos \omega t+y_{2} \sin \omega t, \\
& -\frac{g}{R}\left[\frac{\partial}{\partial \bar{\theta}}(\alpha \eta-\beta \zeta)\right]=z_{1} \cos \omega t+z_{2} \sin \omega t,
\end{aligned}
$$

where $\omega$ is the tidal frequency of interest and $a_{1}, a_{2}, b_{1}, b_{2}, y_{1}, y_{2}, z_{1}$, and $z_{2}$ are constants. The elevation, $\zeta$, and Newton's equilibrium tide, $\eta$, are periodic with frequencies shown in Table A.1. The $y$ 's and $z$ 's are computed from (A.2) by expanding known functions, $\zeta$ and $\eta$, in terms of sines and cosines. Substituting (A.1) and (A.2) in (3.2) leads to four linear equations, in the unknowns $a_{1}, a_{2}, b_{1}$, and $b_{2}$, which can be written in matrix form as,

$$
\left(\begin{array}{cccc}
0 & -2 \Omega \cos \bar{\theta} & -\omega & k_{d} \\
-\omega & k_{d} & 0 & 2 \Omega \cos \bar{\theta} \\
-2 \Omega \cos \bar{\theta} & 0 & k_{d} & \omega \\
k_{d} & \omega & 2 \Omega \cos \bar{\theta} & 0
\end{array}\right)\left(\begin{array}{c}
a_{1} \\
a_{2} \\
b_{1} \\
b_{2}
\end{array}\right)=\left(\begin{array}{c}
y_{2} \\
z_{2} \\
y_{1} \\
z_{1}
\end{array}\right) .
$$




\begin{tabular}{ccrrrr}
\hline \hline \multicolumn{2}{c}{ Semidiurnal Modes } & & \multicolumn{2}{c}{ Diurnal Modes } \\
\cline { 1 - 2 } \cline { 5 - 6 } Component & $\begin{array}{c}\text { Frequency } \\
\text { (cpd) }\end{array}$ & & Component & $\begin{array}{c}\text { Frequency } \\
\text { (cpd) }\end{array}$ \\
\cline { 1 - 2 } \cline { 5 - 6 } M2 & 1.93227 & & K1 & 1.00273 \\
S2 & 2.00000 & & O1 & 0.92954 \\
N2 & 1.89600 & & P1 & 0.99726 \\
K2 & 2.00547 & & Q1 & 0.89325 \\
\hline
\end{tabular}

Table A.1: Major Tidal Frequencies

The solution to this matrix equation determines $u$ and $v$.

We use the values,

$$
\alpha=.69, \quad \beta=.90 \text {, }
$$

derived by Schwiderski [1978], and the value of the linear damping coefficient, $k_{d}$, is selected by minimizing the phase difference between predictions of $\Delta T\left(t, M 2, \Gamma_{R 3}\right)$ using Schwiderski [1983] and damped LTE currents. The resulting best value is,

$$
k_{d}=9.0 \times 10^{-6} \mathrm{~s}^{-1} \text {. }
$$

The value of $k_{d}$ also controls the $Q$ of the LTE model. We estimate $Q$ by comparing the equations of motion to a forced linear-damped oscillator,

$$
\frac{\partial^{2} x}{\partial t^{2}}+k \frac{\partial x}{\partial t}+\omega_{0}^{2} x=\mathrm{A} \cos \omega t
$$

whose $Q$ is defined as,

$$
Q \equiv \frac{\omega_{\mathrm{R}}}{k}
$$

where the resonant frequency is,

$$
\omega_{\mathrm{R}}=\left(\omega_{0}^{2}-\frac{k^{2}}{2}\right)^{1 / 2}
$$

[Marion, 1970]. By letting east be the $x$-direction, and substituting (A.2) into (3.2), we obtain the following $x$-direction momentum equation,

$$
\frac{\partial^{2} x}{\partial t^{2}}+k_{d} \frac{\partial x}{\partial t}-f v=y_{1} \cos \omega t+y_{2} \sin \omega t
$$


where $f=2 \Omega \cos \bar{\theta}$ is the inertial frequency. Since,

$$
\begin{array}{ll}
\left|\frac{\partial u}{\partial t}\right| & \approx 1.4 \times 10^{-6}, \\
|f v| & \approx 1.4 \times 10^{-6}, \\
\left(y_{1}^{2}+y_{2}^{2}\right)^{1 / 2} & \approx 4.4 \times 10^{-7}, \\
\left|k_{d} u\right| & \approx 9.0 \times 10^{-8},
\end{array}
$$

the dominant terms in (A.9) can be written

$$
\frac{\partial u}{\partial t}-f v=0
$$

Substituting the solution to (A.11),

$$
v=\frac{i \omega u}{f}
$$

and the relation,

$$
u=i \omega x,
$$

into (A9), we obtain the approximation,

$$
\frac{\partial^{2} x}{\partial t^{2}}+k_{d} \frac{\partial x}{\partial t}+\omega^{2} x \simeq y_{1} \cos \omega t+y_{2} \sin \omega t .
$$

Comparing (A.6,7,8) with (A.14) we see,

$$
Q \approx \frac{\left(\omega^{2}-\frac{k_{d}^{2}}{2}\right)^{1 / 2}}{k_{d}} \simeq \frac{\omega}{k_{d}}=\frac{1.41 \times 10^{-4} \mathrm{~s}^{-1}}{9.0 \times 10^{-6} \mathrm{~s}^{-1}}=16,
$$

where $1.41 \times 10^{-4} \mathrm{~s}^{-1}$ is the frequency of the $M 2$ tide. This agrees with estimates ranging from 5 to 25 provided by Hendershott [1981].

A comparison of the damped LTE model with the Schwiderski [1983] model for M2 in the North Pacific ocean demonstrates good agreement (Figure A-1). Both models also generally agree with the data presented by Luyten and Stommel [1990] extracted from deep moored current meter records. By extension, we believe that the damped LTE model should be an accurate first order predictor of the $S 2, N 2$, and $K 2$ semidiurnal currents.

The damped LTE model does not perform as well as the Schwiderski [1983] M2 current model in the vicinity of amphidromic points. The finite difference approximations we use 


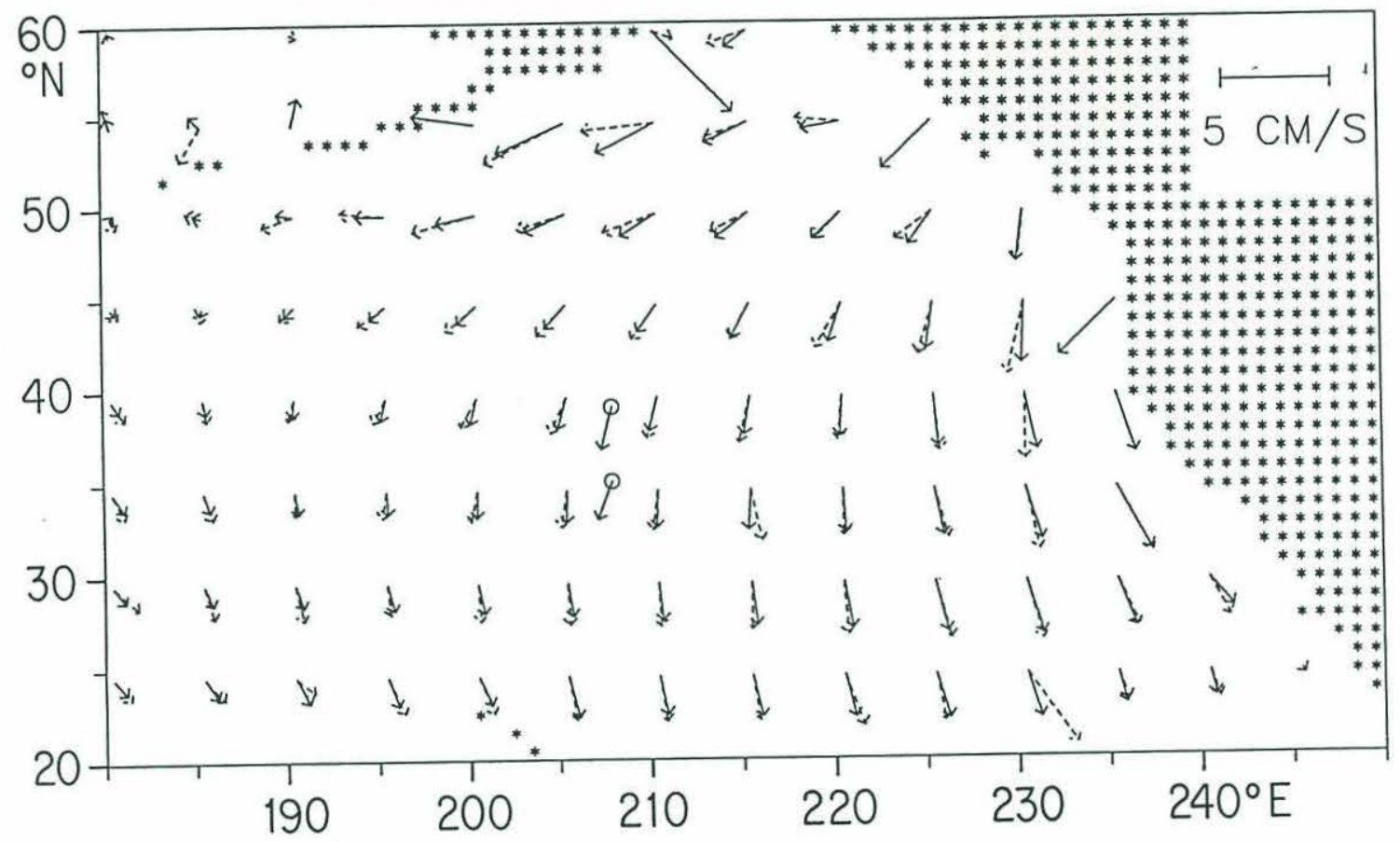

Figure A-1: The $M 2$ barotropic tidal current in the northeast Pacific Ocean at 0600 Greenwich mean time on 1 January 1989 as determined from (a) the Schwiderski [1983] model (solid arrows) (b) linear-damped Laplace tidal equations (Equation (3.2); dashed arrows) (c) data extracted from deep moored current meter records (two arrows designated by circles around their tails). The current meter analysis is taken from Luyten and Stommel [1990] 
for the spatial derivatives in (3.2) are not accurate near these areas of rapidly changing phase. These inaccuracies are limited to a $100 \mathrm{~km}$ (the grid size of Schwiderski's model) radius, and the net effect on predicted travel-time change is less than $5 \%$ in amplitude and 3 degrees in phase for a $4000 \mathrm{~km}$ geodesic passing through an amphidrome.

The damped LTE model has an additional difficulty for diurnal currents within a $\pm 10^{\circ}$ band centered at the inertial latitude,

$$
\theta_{f}=\sin ^{-1}\left(\omega_{k} / 2 \Omega\right)
$$

For diurnal tides, $\omega_{k} \sim 1 \mathrm{cpd}$ and $\theta_{f} \cong 30^{\circ} \mathrm{N}$, and for semidiurnal tides, $\omega_{k} \sim 2 \mathrm{cpd}$ and $\theta_{f} \cong 90^{\circ} \mathrm{N}$. The difficulty can be seen by setting $k_{d}=0$ and solving (3.2) for $u$ and $v$. The resulting equations,

$$
\begin{aligned}
& u=\frac{g\left[-2 \Omega \cos \bar{\theta} \frac{\partial}{\partial \bar{\theta}}(\alpha \eta-\beta \zeta)+\frac{i \omega}{\sin \bar{\theta}} \frac{\partial}{\partial \lambda}(\alpha \eta-\beta \zeta)\right]}{R\left(4 \Omega^{2} \cos ^{2} \bar{\theta}-\omega^{2}\right)}, \\
& v=\frac{g\left[\frac{-2 \Omega}{\tan \bar{\theta}} \frac{\partial}{\partial \lambda}(\alpha \eta-\beta \zeta)-i \omega \frac{\partial}{\partial \bar{\theta}}(\alpha \eta-\beta \zeta)\right]}{R\left(4 \Omega^{2} \cos ^{2} \bar{\theta}-\omega^{2}\right)},
\end{aligned}
$$

have denominators equal to zero at the inertial latitude. These zeros are canceled by numerator zeros when $u, v$, and $\zeta$ are determined exactly from the same equations of motion, but a singularity occurs when the numerators are approximated by finite differences. The finite $k_{d}$ we use in (3.2) removes the singularity in (A.17), but the solution is still inaccurate near $30^{\circ} \mathrm{N}$ (Figure A-2). 


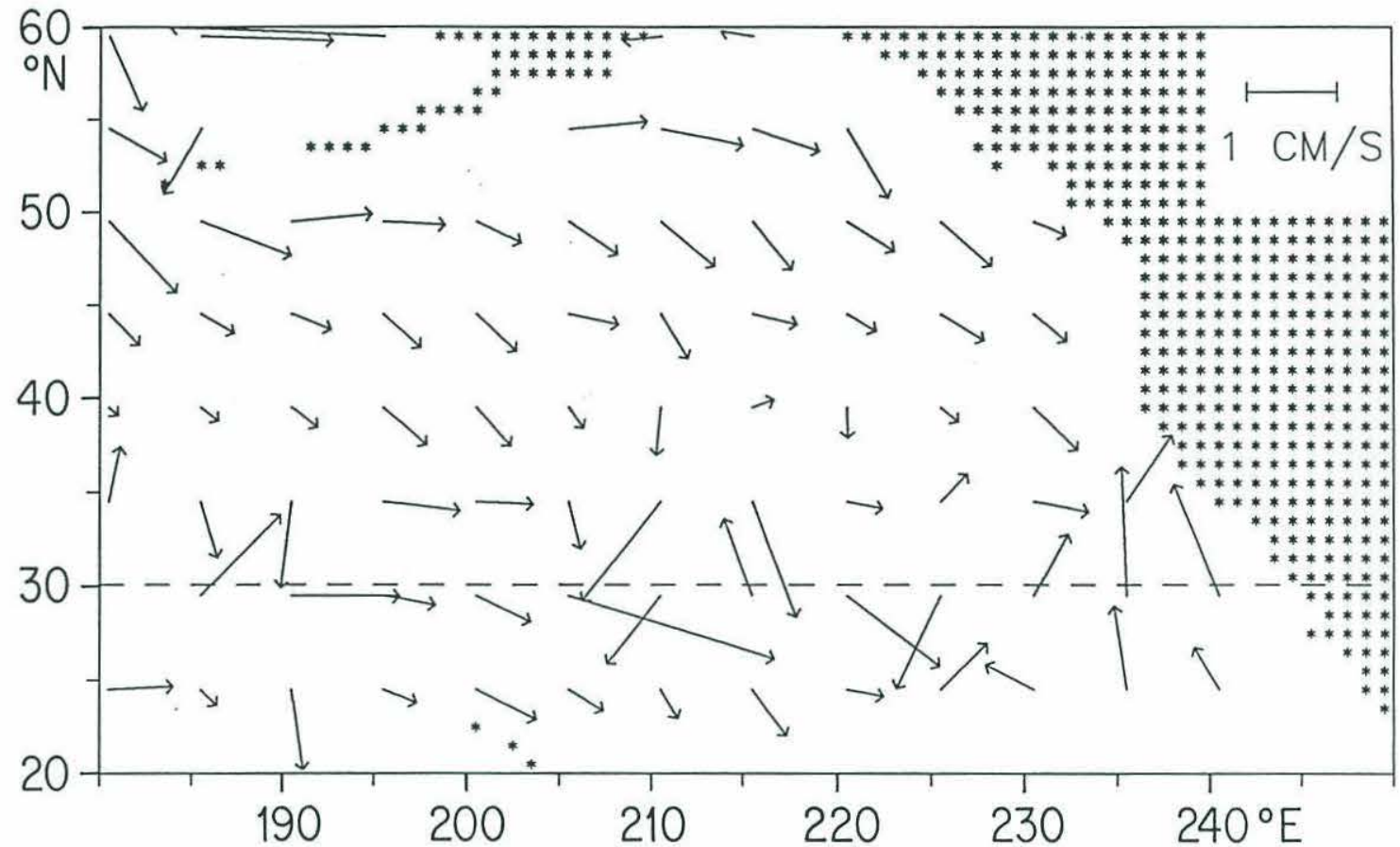

Figure A-2: The $K 1$ barotropic tidal current in the northeast Pacific Ocean at 0600 Greenwich mean time on 1 January 1989 as determined from the linear-damped Laplace tidal equations (Equation (A.2)). Note the irregular performance of the model in the vicinity of $30.1^{\circ} \mathrm{N}$. 


\section{Appendix B}

\section{Guyot-Generated Internal Tides}

In order to determine the coupling between barotropic tidal currents and internal tides generated by guyots, we use the two-dimensional two-layer model illustrated by Figure B1. This model is applicable to sea plateaus whose major horizontal extent is perpendicular to the direction of the barotropic current. We follow the procedure used in deriving the coupling between surface tides and coastal generated internal tides [Rattray, 1960]. Variables are a function only of the distance from the center of the plateau (y-direction) in this two-dimensional model.

A rigid-lid approximation is used. The momentum equations are,

$$
\begin{gathered}
\partial_{t} v^{\prime}+f u^{\prime}=0, \\
\partial_{t} v^{\prime \prime}+f u^{\prime \prime}=-g_{r} \partial_{y} \zeta^{\prime \prime},
\end{gathered}
$$

and,

$$
\begin{gathered}
\partial_{t} u^{\prime}-f v^{\prime}=0, \\
\partial_{t} u^{\prime \prime}-f v^{\prime \prime}=0,
\end{gathered}
$$

where $u$ is the current parallel to the plateau, $\left({ }^{\prime}\right)$ terms correspond to upper layer and (") to lower layer terms, $f$ is the inertial frequency, and,

$$
g_{r}=g \Delta \rho / \rho,
$$

is reduced gravity, where,

$$
\Delta \rho=\rho^{\prime \prime}-\rho^{\prime} .
$$




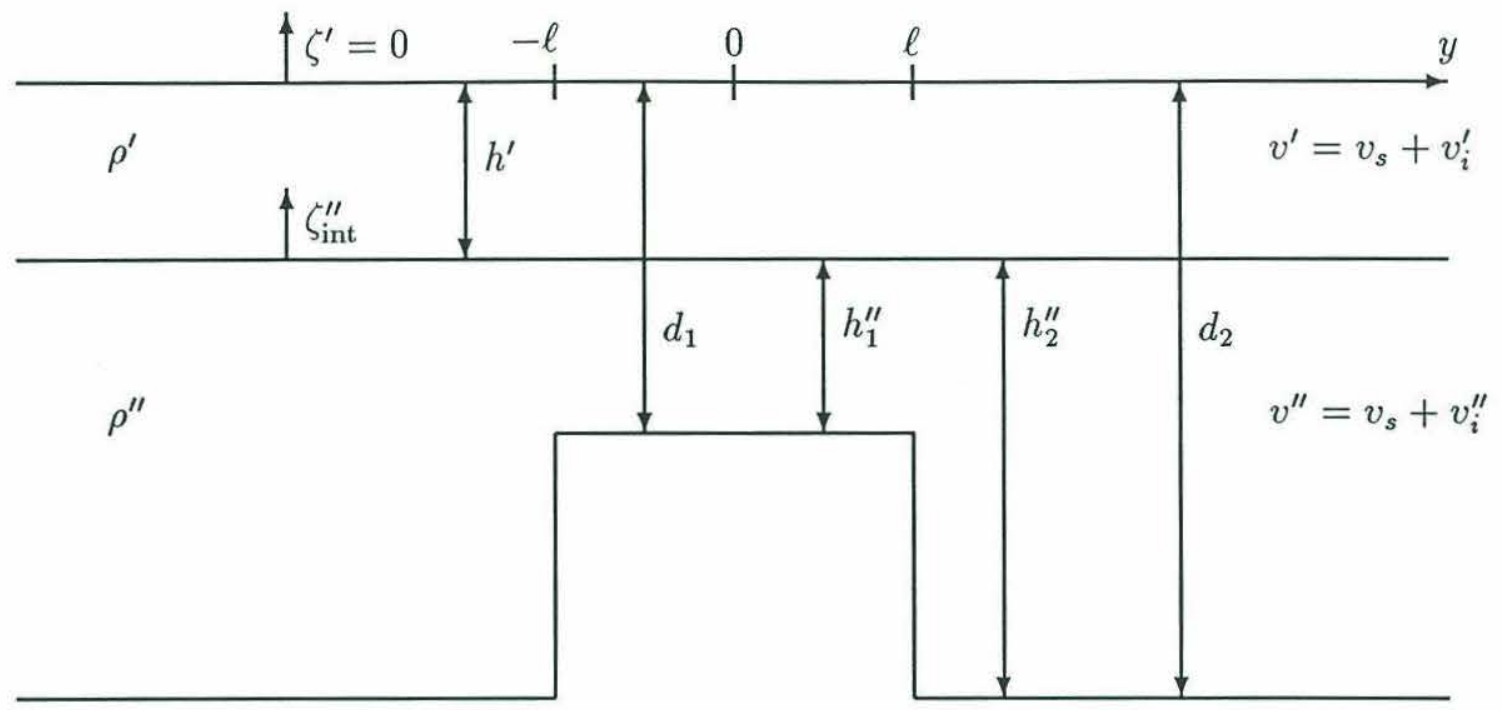

Figure B-1: Definition Figure for a two-layer ocean model used to predict the coupling between a barotropic tidal current and the internal tide generated by a seamount whose extent perpendicular to the direction of the current is considered infinite.

Subtracting (B.2) from (B.1), we find,

$$
\partial_{t}\left(v^{\prime}-v^{\prime \prime}\right)+f\left(u^{\prime}-u^{\prime \prime}\right)=g_{r} \partial_{y} \zeta^{\prime \prime},
$$

and the continuity equations for each layer are,

$$
\begin{aligned}
& \partial_{y}\left(h^{\prime} v^{\prime}\right)=\partial_{t} \zeta^{\prime \prime}, \\
& \partial_{y}\left(h^{\prime \prime} v^{\prime \prime}\right)=-\partial_{t} \zeta^{\prime \prime} .
\end{aligned}
$$

Let $v_{s}=(a / d) \exp i(\omega t+\phi)$, which is essentially constant for any distance y small compared to the wavelength of the surface tide. We have contributions to the currents in the two layers from both the barotropic current and the currents associated with the internal tide. Thus,

$$
\begin{array}{ll}
v^{\prime}=v_{s}+v_{i}^{\prime} & u^{\prime}=u_{s}+u_{i}^{\prime} \\
v^{\prime \prime}=v_{s}+v_{i}^{\prime \prime} & u^{\prime \prime}=u_{s}+u_{i}^{\prime \prime},
\end{array}
$$

and from the rigid-lid approximation,

$$
\begin{aligned}
& \zeta^{\prime}=0, \\
& \zeta^{\prime \prime}=\zeta_{\text {int }}^{\prime \prime} .
\end{aligned}
$$


We choose the following solutions which satisfy (B.3) and (B.7),

$$
\begin{array}{llll} 
& y<-\ell & -\ell<y<\ell & y>\ell \\
\zeta_{\text {int }}^{\prime \prime} & -B e^{i k_{2} y} & A \sin k_{1} y & B e^{-i k_{2} y} \\
v_{s} & 1 / d_{2} & 1 / d_{1} & 1 / d_{2} \\
v_{i}^{\prime} & -\left(\omega / k_{2} h^{\prime}\right) B e^{i k_{2} y} & -\left(i \omega / k_{1} h^{\prime}\right) A \cos k_{1} y & -\left(\omega / k_{2} h^{\prime}\right) B e^{-i k_{2} y} \\
v_{i}^{\prime \prime} & \left(\omega / k_{2} h_{2}^{\prime \prime}\right) B e^{i k_{2} y} & \left(i \omega / k_{1} h_{1}^{\prime \prime}\right) A \cos k_{1} y & \left(\omega / k_{2} h_{2}^{\prime \prime}\right) B e^{-i k_{2} y} \\
u_{s} & -i f / \omega d_{2} & -i f / \omega d_{1} & -i f / \omega d_{2} \\
u_{i}^{\prime} & \left(i f / k_{2} h^{\prime}\right) B e^{i k_{2} y} & -\left(f / k_{1} h^{\prime}\right) A \cos k_{1} y & \left(i f / k_{2} h^{\prime}\right) B e^{-i k_{2} y} \\
u_{i}^{\prime \prime} & -\left(i f / k_{2} h_{2}^{\prime \prime}\right) B e^{i k_{2} y} & \left(f / k_{2} h_{1}^{\prime \prime}\right) A \cos k_{1} y & -\left(i f / k_{2} h_{2}^{\prime \prime}\right) B e^{-i k_{2} y},
\end{array}
$$

with the common multiplier,

$$
a \exp ^{i(\omega t+\phi)} .
$$

To satisfy (B.6),

$$
k_{j}^{2}=\frac{\omega^{2}-f^{2}}{g_{r} h^{\prime} h_{j}^{\prime \prime}} d_{j} ; \quad j=1,2 .
$$

In order to patch the solutions at the edges of the plateau, $\zeta^{\prime \prime}$ must be continuous at $y=\ell$ and $y=-\ell$. Thus,

$$
B e^{-i k_{2} \ell}=A \sin k_{1} \ell .
$$

The solutions (B.10) already satisfy the conservation of total mass flux $h^{\prime} v^{\prime}+h^{\prime \prime} v^{\prime \prime}=$ $d v_{s}+h^{\prime} v_{i}^{\prime}+h^{\prime \prime} v_{i}^{\prime \prime}$ across $y=\ell$ and $y=-\ell$, but conservation of $\rho^{\prime}$ mass flux requires that $h^{\prime} v^{\prime}=h^{\prime}\left(v_{s}+v_{i}^{\prime}\right)$ be continuous also. To satisfy this requirement we let,

$$
\begin{aligned}
& A=A_{0} e^{-i(\alpha-\pi / 2)}, \\
& B=B_{0} e^{-i\left(\alpha-\pi / 2-k_{2} \ell\right)} .
\end{aligned}
$$

Substituting (B.14) into (B.13) leads to,

$$
A_{0} \sin k_{1} \ell=B_{0} \text {. }
$$

Using these relations and conservation of $\rho^{\prime}$ mass flux we find,

$$
\frac{h^{\prime}}{d_{2}}-\frac{\omega}{k_{2}} A_{0}(\sin \alpha+i \cos \alpha) \sin k_{1} \ell=\frac{h^{\prime}}{d_{1}}-\frac{i \omega}{k_{1}} A_{0}(\sin \alpha+i \cos \alpha) \cos k_{1} \ell .
$$

By equating the imaginary part of (B.16) we obtain,

$$
\tan \alpha=\frac{k_{1}}{k_{2}} \tan k_{1} \ell,
$$




\begin{tabular}{|c|c|c|c|c|}
\hline \multirow[b]{3}{*}{$\zeta_{\text {int }}^{\prime \prime}$} & \multicolumn{2}{|c|}{$|y|<\ell$} & \multicolumn{2}{|r|}{$|y|>\ell$} \\
\hline & Amplitude & $\operatorname{Phase}\left(^{\circ}\right)$ & Amplitude & $\operatorname{Phase}\left({ }^{\circ}\right)$ \\
\hline & $a A_{0} \sin k_{1} y$ & $\phi-\alpha+90$ & $a B_{0}(|y| / y)$ & $\phi-\alpha+90-k_{2}(|y|-\ell)$ \\
\hline$v_{s}$ & $a / d_{1}$ & $\phi$ & $a / d_{2}$ & $\phi$ \\
\hline$v_{i}^{\prime}$ & $a \omega A_{0} \cos k_{1} y / k_{1} h^{\prime}$ & $\phi-\alpha$ & $a \omega B_{0} / k_{2} h^{\prime}$ & $\phi-\alpha+270-k_{2}(|y|-\ell)$ \\
\hline$v_{i}^{\prime \prime}$ & $a \omega A_{0} \cos k_{1} y / k_{1} h_{1}^{\prime \prime}$ & $\phi-\alpha+180$ & $a \omega B_{0} / k_{2} h_{2}^{\prime \prime}$ & $\phi-\alpha+90-k_{2}(|y|-\ell)$ \\
\hline$u_{s}$ & $a f / \omega d_{1}$ & $\phi+270$ & $a f / \omega d_{2}$ & $\phi+270$ \\
\hline$u_{i}^{\prime}$ & af $A_{0} \cos k_{1} y / k_{1} h^{\prime}$ & $\phi-\alpha+270$ & af $B_{0} / k_{2} h^{\prime}$ & $\phi-\alpha+180-k_{2}(|y|-\ell)$ \\
\hline$u_{i}^{\prime \prime}$ & af $A_{0} \cos k_{1} y / k_{1} h_{1}^{\prime \prime}$ & $\phi-\alpha+90$ & $a f B_{0} / k_{2} h_{2}^{\prime \prime}$ & $\phi-\alpha-k_{2}(|y|-\ell)$ \\
\hline
\end{tabular}

Table B.1: Generalized two-layer internal wave solution for a barotropic north-south tidal current propagating over an undersea plateau of infinite east-west extent.

and by equating the real part, we can write,

$$
A_{0}=\left(\frac{1}{d_{2}}-\frac{1}{d_{1}}\right) \frac{k_{1} h^{\prime} \cos \alpha}{\omega \cos k_{1} \ell},
$$

which leads to,

$$
B_{0}=\left(\frac{1}{d_{2}}-\frac{1}{d_{1}}\right) \frac{k_{2} h^{\prime}}{\omega} \sin \alpha .
$$

The result of this solution is a standing internal wave over the extent of the plateau which patches to traveling internal waves at either end. The generalized solution is collected in Table B.1, and the particular solution for a guyot located at $30.3^{\circ} \mathrm{N}, 222.5^{\circ} \mathrm{E}$ is summarized in Table B.2.

The cycle-average baroclinic energy density for this two-layer ocean is,

$$
\overline{E_{i}}=\frac{1}{\mathrm{~T}} \int_{0}^{\mathrm{T}} \frac{1}{2} \rho\left[\left(\zeta_{\text {int }}\right)^{2} g_{r}+h^{\prime}\left(u_{i}^{\prime}\right)^{2}+h^{\prime}\left(v_{i}^{\prime}\right)^{2}+h^{\prime \prime}\left(u_{i}^{\prime \prime}\right)^{2}+h^{\prime \prime}\left(v_{i}^{\prime \prime}\right)^{2}\right] d t,
$$

where $\mathrm{T}$ is the period of the internal tide. Substituting the Table B.1 amplitudes and phases in (B.20) results in,

$$
\overline{E_{i}}=\frac{1}{2} \rho g_{r}\left(v_{s} d_{2} B_{0}\right)^{2}\left(\frac{\omega^{2}}{\omega^{2}-f^{2}}\right),
$$

where the resulting energy density is in joules per square meter. This baroclinic energy propagates at a group speed that from (B.12) is given by,

$$
c_{g i}=\frac{\omega^{2}-f^{2}}{k_{2} \omega} .
$$




\begin{tabular}{|c|c|c|c|c|}
\hline \multirow[b]{3}{*}{ Constituient } & \multicolumn{2}{|c|}{$M 2$} & \multicolumn{2}{|c|}{$S 2$} \\
\hline & & Greenwich & & Greenwich \\
\hline & Amplitude & $\operatorname{Phase}\left({ }^{\circ}\right)$ & Amplitude & $\operatorname{Phase}\left({ }^{\circ}\right)$ \\
\hline$\zeta_{\text {int }}^{\prime \prime}$ & $6.21 \mathrm{~m}$ & $166+k_{2} y$ & $2.08 \mathrm{~m}$ & $202+k_{2} y$ \\
\hline$v_{s}$ & $2.3 \mathrm{~cm} \cdot \mathrm{s}^{-1}$ & 68 & $0.7 \mathrm{~cm} \cdot \mathrm{s}^{-1}$ & 104 \\
\hline$v_{i}^{\prime}$ & $1.9 \mathrm{~cm} \cdot \mathrm{s}^{-1}$ & $346+k_{2} y$ & $0.6 \mathrm{~cm} \cdot \mathrm{s}^{-1}$ & $22+k_{2} y$ \\
\hline$v_{i}^{\prime \prime}$ & $0.3 \mathrm{~cm} \cdot \mathrm{s}^{-1}$ & $166+k_{2} y$ & $0.1 \mathrm{~cm} \cdot \mathrm{s}^{-1}$ & $202+k_{2} y$ \\
\hline$u_{s}$ & $1.2 \mathrm{~cm} \cdot \mathrm{s}^{-1}$ & 158 & $0.4 \mathrm{~cm} \cdot \mathrm{s}^{-1}$ & 194 \\
\hline$u_{i}^{\prime}$ & $1.0 \mathrm{~cm} \cdot \mathrm{s}^{-1}$ & $76+k_{2} y$ & $0.3 \mathrm{~cm} \cdot \mathrm{s}^{-1}$ & $112+k_{2} y$ \\
\hline$u_{i}^{\prime \prime}$ & $0.2 \mathrm{~cm} \cdot \mathrm{s}^{-1}$ & $256+k_{2} y$ & $0.1 \mathrm{~cm} \cdot \mathrm{s}^{-1}$ & $292+k_{2} y$ \\
\hline
\end{tabular}

Table B.2: Two-layer $M 2$ and $S 2$ internal tide solutions for regions north of a 22 by 111 $\mathrm{km}$ undersea plateau. Seamount is located at $30.3^{\circ} \mathrm{N}, 222.5^{\circ} \mathrm{E}$ (Figure 1-1), $\ell=11 \mathrm{~km}$, $d_{1}=2200 \mathrm{~m}, d_{2}=4700 \mathrm{~m}, h^{\prime}=700 \mathrm{~m}, g_{r}=.0059 \mathrm{~m} \cdot \mathrm{s}^{-2}, k_{2}(\mathrm{M} 2)=6.41 \times 10^{-5} \mathrm{~m}^{-1}$ (wavelength is $98 \mathrm{~km}$ ), and $k_{2}(\mathrm{~S} 2)=6.71 \times 10^{-5} \mathrm{~m}^{-1}$ (wavelength is $94 \mathrm{~km}$ ).

The group speed, energy density, and horizontal extent of each seamount are combined in (4.4) to obtain the barotropic-to-baroclinic tidal energy conversion rate. Expansion of (4.4) in terms of fundamental parameters results in,

$$
\frac{d E}{d t}=\frac{\Delta x}{\omega} \rho\left(h^{\prime}\right)^{3 / 2}\left(d_{2} g_{r}\right)^{1 / 2} v_{s}^{2}\left(1-\frac{d_{1}}{d_{2}}\right)^{2}\left(\frac{\omega^{2}-f^{2}}{h_{2}^{\prime \prime}}\right)^{1 / 2} \sin ^{2} \alpha,
$$

where,

$$
\tan \alpha=\left(\frac{d_{1} h_{2}^{\prime \prime}}{d_{2} h_{1}^{\prime \prime}}\right)^{1 / 2} \tan \left[\left(\frac{\omega^{2}-f^{2}}{g_{r} h^{\prime} h_{1}^{\prime \prime}}\right)^{1 / 2} \ell\right] .
$$

The internal tide in Table B.2 has an energy density and group speed of about $1.5 \times 10^{5}$ $\mathrm{erg} \cdot \mathrm{cm}^{-2}$ and $160 \mathrm{~cm} \cdot \mathrm{s}^{-1}$ respectively. The energy density near this seamount is about $17 \%$ of that contained in the parent barotropic tide, and the baroclinic conversion rate is about $5.3 \times 10^{14} \mathrm{erg} \cdot \mathrm{s}^{-1}(53 \mathrm{Mw})$.

The reduced gravity (B.4) used in the dispersion relation (B.12) is subject to the choices for the representative densities $\rho^{\prime}$ and $\rho^{\prime \prime}$ in the upper and lower layers. Values of $g_{r}$ of order $10^{-2} \mathrm{~m} \cdot \mathrm{s}^{-2}$ are typical for two-layer ocean models, but using $g_{r}=10^{-2} \mathrm{~m} \cdot \mathrm{s}^{-2}$ is not optimal for minimizing the mean square error between the combined model and the measured travel time signals at R4. The optimal value of reduced gravity chosen for this model, as illustrated by Figure B2, is about $5.9 \times 10^{-3} \mathrm{~m} \cdot \mathrm{s}^{-2}$. 


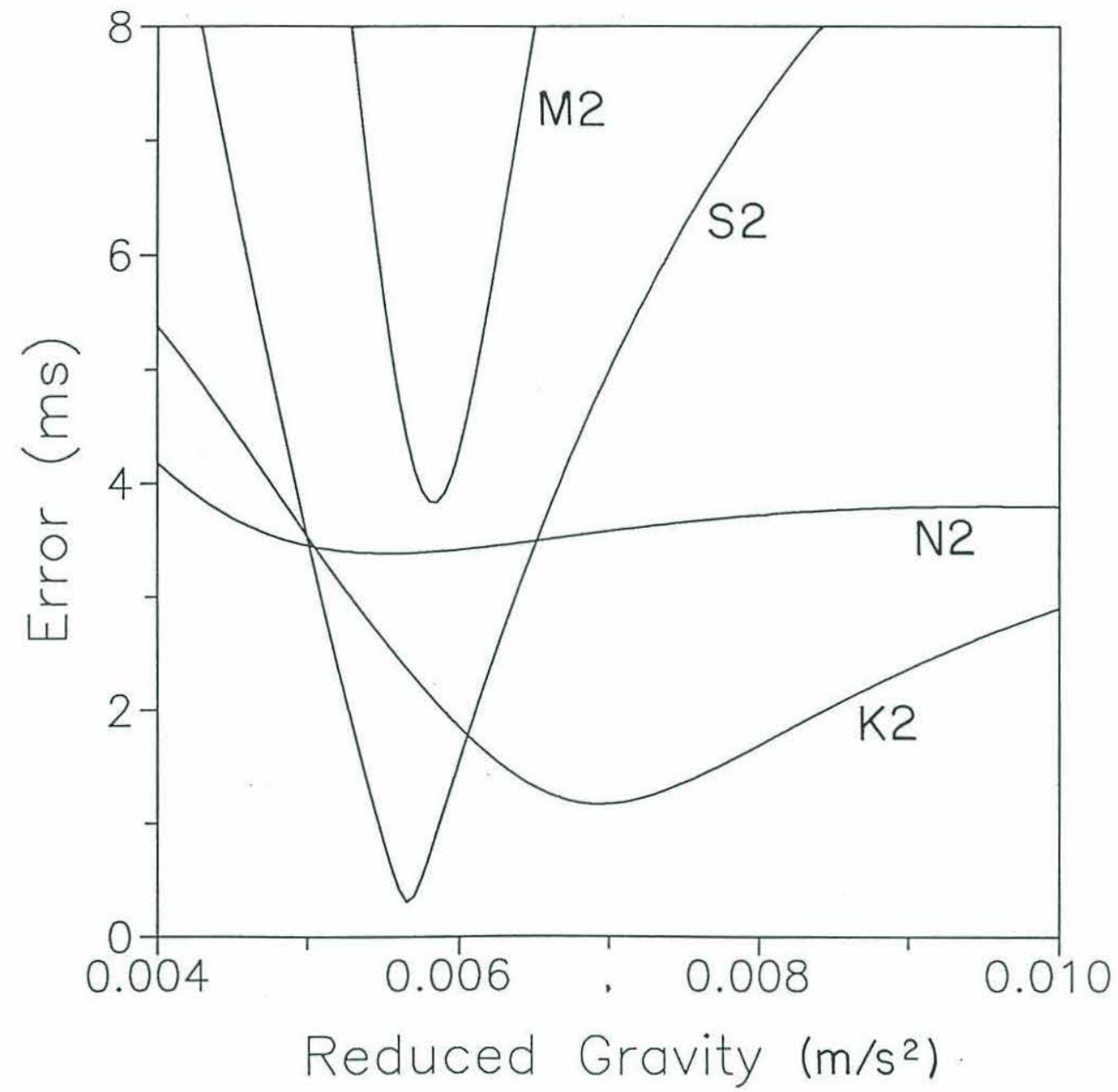

Figure B-2: Magnitude of Receiver 4 barotropic plus baroclinic signal prediction errors. The errors are plotted as a function of reduced gravity. 


\section{Appendix C}

\section{Baroclinic Tide Travel-Time}

\section{Model}

Equation (3.5) predicts the travel-time modulation of a single multipath caused by one seamount. The multipaths oscillate about the sound axis, and a typical wavelength (upper plus lower loop) is $50 \mathrm{~km}$. The potential sound speed gradient in the region of the Table B.2 plateau is approximately,

$$
\frac{\partial c_{p}}{\partial z} \simeq \begin{cases}0.13 \mathrm{~s}^{-1} & \text { above axis } \\ 0.0 \mathrm{~s}^{-1} & \text { below axis }\end{cases}
$$

where positive $z$ is upward. We assume the average ray path is above axis for $50 \%$ of its travel adjacent to the $111 \mathrm{~km}$ length of the plateau, so the average change in travel-time at the $M 2$ period is,

$$
\Delta T_{\mathrm{bc}}(t)=\frac{\left(6.2 e^{\left.i \omega\left(t-\frac{l}{c_{0}}\right)+\theta_{\zeta_{\text {int }}} \mathrm{m}\right)\left(0.13 \mathrm{~s}^{-1}\right)(111 \mathrm{~km})}\right.}{(2)\left(1479 \mathrm{~m} \mathrm{~s}^{-1}\right)^{2}}=21 e^{i \omega\left(t-\frac{l}{c_{0}}\right)+\theta_{\zeta_{\text {int }}} \mathrm{ms}},
$$

where $\theta_{\text {Sint }}$ is the phase of the $M 2$ internal tide, and $\omega$ is the $M 2$ frequency.

An evaluation of $N_{s}$ seamount-generated tides along the geodesic between source and receiver will produce $N_{s}$ baroclinic signals at each tidal frequency. The vector sum of baroclinic models plus the barotropic model results in the combined model,

$$
\Delta T_{\mathrm{tot}}\left(t, \omega_{k}\right)=\Delta T_{\mathrm{bt}}\left(t, \omega_{k}\right)+\sum_{n=1}^{N_{s}} \Delta T_{\mathrm{bc}}\left(n, t, \omega_{k}\right),
$$


where $\Delta T_{\mathrm{bc}}\left(n, t, \omega_{k}\right)$ is the baroclinic signal prediction for the $n$th seamount at frequency $\omega_{k}$. 


\section{Appendix D}

\section{Signal Processing}

\section{D.1 The Transmitted Signal}

Pulse compression techniques described by Spiesberger et al. [1980] and Metzger [1983] are utilized to increase signal-to-noise ratio without degrading the phase difference resolution. The transmitted signal has a center frequency of $133 \mathrm{~Hz}$ and a bandwidth of about $17 \mathrm{~Hz}$. The center frequency is phase modulated every 8 cycles using a 511 digit maximal shift register sequence having a period of $511(8 / 133) \approx 30.7 \mathrm{~s}$ [Spiesberger et al., 1989a]. The ideal transmitted waveform is,

$$
s(t)=s_{0} \cos \left[2 \pi f_{c} t-\frac{\pi}{2}\left(\mathrm{r}_{k(t)}-\frac{1}{2}\right)\right]
$$

[Spiesberger et al., 1980], where $f_{c}$ is the center frequency, $\mathrm{r}_{k}$ represents the shift register sequence of ones and zeros, and,

$$
k(t)=\sum_{l=0}^{\infty} \mathrm{u}\left(t-\frac{8 l}{133}\right),
$$

where $u(t)$ is the unit step function. 


\section{D.2 Post-Detection Processing}

\section{D.2.1 Demodulation and Averaging}

The received signal, $z(t)$, can be approximated by,

$$
z(t)=\sum_{n=1}^{N_{m p}} b_{n} s\left(t-\tau_{n}\right),
$$

which is the weighted sum of $N_{m p}$ multipaths each having gain $b_{n}$ and delay $\tau_{n}$ [Spiesberger et al., 1980]. Demodulation of the received signal is achieved by sampling at $4 \cdot 133 \mathrm{~Hz}=$ $532 \mathrm{~Hz}$. Samples of an ideal single-path arrival are,

$$
z[\mathrm{n}]=\cos \left(\frac{\mathrm{n} \pi}{2}\right) \cos \left[\frac{\pi}{2}\left(\mathrm{r}_{k[\mathrm{n}]}-1 / 2\right)\right]+\sin \left(\frac{\mathrm{n} \pi}{2}\right) \sin \left[\frac{\pi}{2}\left(\mathrm{r}_{k[\mathrm{n}]}-1 / 2\right)\right],
$$

where,

$$
k[\mathrm{n}]=\sum_{l=0}^{\infty} \mathrm{u}[\mathrm{n}-32 l],
$$

and $u[n]$ is the unit step sequence. Because the sampling frequency is four times the carrier frequency, the even numbered samples are the cosine of the demodulated phase angle (weighted by \pm 1 ) and the odd numbered samples are the sine of the demodulated phase angle (weighted by \pm 1 ). The demodulated phase angles are low-pass filtered by block averaging to achieve a sequence of complex demodulates defined as,

$$
c[j]=x[j]+i y[j] ; j=0,1, \ldots, J-1,
$$

where the square root of minus one equals $i$,

$$
x[j]=\sum_{m=0}^{3}(-1)^{m+j} z[2(m+4 j)],
$$

and,

$$
y[j]=\sum_{m=0}^{3}(-1)^{m+j} z[2(m+4 j)+1],
$$

[Spiesberger et al., 1980]. The resulting sample rate is four complex demodulates per digit, so there are $J=4 \cdot 511=2044$ complex demodulates per sequence. The complex demodulates are averaged over 6 consecutive sequences in 1988 resulting in one averaged sequence record every 184 seconds. The demodulates are averaged over 4 sequences in 1989. The signal-to-noise ratio increases by $10 \log _{10} 6=8 \mathrm{~dB}$ following the six-period average in 1988 and by $10 \log _{10} 4=6 \mathrm{~dB}$ following the four-period average in 1989 . 


\section{D.2.2 Replica Correlation}

One period of a $66.5 \mathrm{~Hz}$ sampling of (D.1) phase angles (expressed as complex demodulates) is contained in the following sequence of length $\mathrm{J}$,

$$
s_{\phi}[j]=\cos \left[\frac{\pi}{2}\left(\mathrm{r}_{k[j]}-\frac{1}{2}\right)\right]+i \sin \left[\frac{\pi}{2}\left(\mathrm{r}_{k[j]}-\frac{1}{2}\right)\right] ; j=0,1, \ldots, J-1,
$$

where,

$$
k[j]=\sum_{l=0}^{\infty} \mathrm{u}[j-4 l],
$$

and $\mathrm{u}[j]$ is the unit step sequence. The received complex demodulates (D.6) are circularly convolved with a sequence-reversed (matched filter) replica of the transmitted demodulates (D.9). The pulse-compressed output is given by,

$$
d[m]=\sum_{j=0}^{J-1} c[j] \tilde{s}_{\phi}^{*}[j+m] ; m=0,1, \ldots, J-1,
$$

where the periodic sequence, $\tilde{s}_{\phi}[j]$, is given by,

$$
\tilde{s}_{\phi}[j]=\sum_{r=-\infty}^{\infty} s_{\phi}[j+r J],
$$

[Oppenheim and Schafer, 1989], and $s_{\phi}^{*}$ is the complex conjugate of $s_{\phi}$. The replica correlation (D.11) compresses $30.7 \mathrm{~s}$ of energy along each multipath into a single pulse of one digit length ( $0.06 \mathrm{~s})$ [Spiesberger et al., 1989a], whose amplitude and location depend on the gain, $b_{n}$, and delay, $\tau_{n}$, in (D.3). The signal-to-noise ratio increases by $10 \log _{10} 511=27 \mathrm{~dB}$ following pulse compression, and a sliding average of 4 complex samples (separated by $0.015 \mathrm{~s}$ ) increases the signal-to-noise ratio by another $6 \mathrm{~dB}$ at the cost of some resolution [Spiesberger et al., 1989a].

\section{D.2.3 Phase Change Between Sequence Records}

The change in phase of the mth pulse-compressed demodulate from record $r-1$ to $r$ is given by the phase of,

$$
d[m, r-1] d^{*}[m, r] .
$$

The average change in phase from record $r-1$ to $r$ is given by the phase of

$$
A e^{i \delta \phi[r]} \equiv \sum_{m=0}^{M} d[m, r-1] d^{*}[m, r],
$$


where there are $M$ demodulates containing a signal [Spiesberger et al., 1989a]. The phase in (D.13) is referred to as the cross-correlated phase, because it is the phase of the complex cross correlation of adjacent pulse-compressed records at zero-lag. The cross-correlated phase can be converted to the average travel-time difference between records using (2.1). The precision of the resulting estimation of travel-time difference is about $135 \mu$ s [Spiesberger et al., 1989a]. 


\section{Bibliography}

Bell, T. H., Topographically generated internal waves in the open ocean, J. Geophys. Res., 80, 320-327, 1975.

Christodoulidis, D. C., D. E. Smith, , R. G. Williamson, and S. M. Klosko, Observed tidal braking in the Earth/Moon/Sun system, J. Geophys. Res., 93, 6216-6236, 1988.

Craig, C. H., and D. T. Sandwell, Global distribution of seamounts from Seasat profiles, J. Geophys. Res., 93, 10,408-10,420, 1988.

Headrick, R. H., J. L. Spiesberger, and P. J. Bushong, Basin-scale acoustic tomography: observations of baroclinic tides generated by seamounts, J. Geophys. Res., (submitted 1990).

Hendershott, M. C., Long waves and ocean tides, Evolution of Physical Oceanography, B. A. Warren and C. Wunsch, Eds. MIT Press, 292-341, 1981.

Jordan, T. H., H. W. Menard, and D. K. Smith, Density and size distribution of seamounts in the eastern Pacific inferred from wide-beam sounding data, J. Geophys. Res., 88, 10,508-10,518, 1983.

Lambeck, K., Tidal dissipation in the oceans, Phil. Trans. R. Soc., 287A, 545-592, 1977.

Lambeck, K., The Earth's variable rotation: geophysical causes and consequences, 449pp., Cambridge University Press, New York, 1980.

Lawson, C. L., and R. J. Hanson, Solving least-squares problems, 340pp., Prentice-Hall, Inc., Englewood Cliffs, NJ., 1974.

Luyten, J. R., and H. M. Stommel, Comparison of M2 tidal currents observed by some deep moored current meters with those of the Schwiderski model, Deep-Sea Res., 1990 (in press).

Marion, J. B., Classical Dynamics of Particles and Systems, 187pp., Academic Press, New York, NY, 1970.

Metzger, K., Signal processing equipment and techniques for use in measuring ocean acoustic multipath structures, Ph.D. thesis, Univ. of Michigan, Ann Arbor, 1983.

Munk, W. H., Abyssal recipes, Deep-Sea Res., 13, 707-730, 1966.

Munk W., B. Zetler, J. Clark, S. Gill, D. Porter, J. Spiesberger, and R. Spindel, Tidal effects on long-range sound transmission, J. Geophys. Res., 86, 6399-6410, 1981b. 
Noble, M., D. A. Cacchione, and W. C. Schwab, Observations of strong mid-Pacific internal tides above Horizon Guyot, J. Phys. Oceanogr., 18, 1300-1306, 1988.

Oppenheim, A. V., and R. W. Schafer, Discrete-time signal processing, 879pp., Prentice Hall, Inc., Englewood Cliffs, NJ, 1989.

Rattray, M., Jr., On the coastal generation of internal tides, Tellus, 12, 54-62, 1960.

Schwiderski, E. W., Global ocean tides, part I: a detailed hydrodynamical interpolation model, Naval Surface Weapons Center, TR 3866, Dahlgreen, Virginia, 88 pp., 1978.

Schwiderski, E. W., Global ocean tides, part II: the semidiurnal principal lunar tide (M2), atlas of tidal charts and maps, Naval Surface Weapons Center, TR 79-414, Dahlgreen, Virginia, 15pp., 1979.

Schwiderski, E. W., Global ocean tides, part III: the semidiurnal principal solar tide (S2), atlas of tidal charts and maps, Naval Surface Weapons Center, TR 81-122, Dahlgreen, Virginia, 12pp., 1981.

Schwiderski, E. W., Global ocean tides, part VI: the semidiurnal elliptical lunar tide (N2), atlas of tidal charts and maps, Naval Surface Weapons Center, TR 81-218, Dahlgreen, Virginia, 11pp., 1981.

Schwiderski, E. W., Global ocean tides, part VIII: the semidiurnal luni-solar declination tide (K2), atlas of tidal charts and maps, Naval Surface Weapons Center, TR 81-222, Dahlgreen, Virginia, 11pp., 1981.

Schwiderski, E. W., Global ocean tides, part IV: the diurnal luni-solar declination tide (K1), atlas of tidal charts and maps, Naval Surface Weapons Center, TR 81-142, Dahlgreen, Virginia, 12pp., 1981.

Schwiderski, E. W., M2 global ocean tide velocity tape, Naval Surface Weapons Center, Dahlgreen, Virginia, 1983.

Smith, D. K., Shape Analysis of Pacific Seamounts, Earth Planet Sci. Lett., 90, 457466, 1988.

Spiesberger, J. L., R. C. Spindel, K. Metzger, Stability and indentification of ocean acoustic multipaths, J. Acoust. Soc. Am., 67, 2011-2017, 1989b.

Spiesberger, J. L., P. J. Bushong, K. Metzger, and T. G. Birdsall, Ocean acoustic tomography: estimating the acoustic travel-time with phase, IEEE J. Oceanic Eng., 14, 108-119, 1989a. 
Spiesberger, J. L., P. J. Bushong, K. Metzger, and T. G. Birdsall, Basin-scale tomography: synoptic measurements of a $4000 \mathrm{~km}$ length section in the Pacific, J. Phys. Oceanogr., 19, 1073-1090, 1989b.

Worcester, P. F., B. D. Dushaw, and B. M. Howe, Gyre-scale current measurements using reciprocal acoustic transmissions Proceedings of the Fourth IEEE Working Conference on Current Measurement, Clinton, MD, April 3-5, 1990 (in press).

Wunsch C., Internal Tides in the Ocean, Rev. Geophys. Space Physics, 13, 167-182, 1975. 